\title{
Adaptive Control of Teleoperation Systems with Linearly and Nonlinearly Parameterized Dynamic Uncertainties
}

\author{
$\mathrm{Xia} \mathrm{Liu}^{1,2}$ and Mahdi Tavakoli ${ }^{2}$ \\ ${ }^{1}$ School of Automation Engineering, University of Electronic Science and Technology of China, \\ Chengdu, Sichuan, China 611731 \\ ${ }^{2}$ Department of Electrical and Computer Engineering, University of Alberta, \\ Edmonton, Alberta, Canada T6G 2V4 \\ Email: xia8@ualberta.ca, tavakoli@ece.ualberta.ca
}

\begin{abstract}
Existing work concerning adaptive control of uncertain teleoperation systems only deals with linearly parameterized (LP) dynamic uncertainties. Typical teleoperation system dynamics, however, also posses terms with nonlinearly parameterized (NLP) structures. An example of such terms is friction, which is ubiquitous in the joints of the master and slave robots of practical teleoperation systems. Uncertainties in the NLP dynamic terms may lead to significant position and force tracking errors if not compensated for in the control scheme. In this paper, adaptive controllers are designed for the master and slave robots with both LP and NLP dynamic uncertainties. Next, these controllers are incorporated into the 4-channel bilateral teleoperation control framework to achieve transparency. Then, transparency of the overall teleoperation is studied via a Lyapunov function analysis. Simulation studies demonstrate the effectiveness of the proposed adaptive scheme when exact knowledge of the LP and NLP dynamics is unavailable.
\end{abstract}

Keywords: Teleoperation system, LP dynamic uncertainty, NLP dynamic uncertainty, adaptive control, transparency

\section{Introduction}

Teleoperation systems offer humans the ability to interact with environments that are inaccessible. They 
have been widely applied in areas such as outer space and undersea exploration [1-2] control of construction/forestry machines of the excavator type [3], minimally invasive telesurgery [4], nuclear waste site and radioactive material management [5], and so on.

The block diagram of a general bilateral teleoperation system is shown in Fig. 1, where $\mathbf{x}_{m}$ and $\mathbf{x}_{s}$ are the positions of the master and the slave, respectively, $\mathbf{f}_{h}$ is the force that the operator applies to the master, and $\mathbf{f}_{e}$ is the force that the environment applies to the slave. The human operator applies forces on the master to remotely control the position of the slave in order to perform a task. If the slave robot exactly reproduces the master's position trajectory and the master robot accurately reproduces the slave-environment contact force to the human operator, the teleoperation system is said to be fully transparent.

For precise teleoperation, transparency is essential. In order to ensure transparency, various control approaches have been proposed for teleoperation systems [6-11]. Among these control approaches, the 4channel architecture is the most successful in terms of fulfilling transparency [12-13]. However, this control scheme assumes perfect knowledge of the linear impedances of the master and the slave. In other words, these controllers are fixed. Nevertheless, in practice, the exact knowledge of the master and the slave dynamic models may be unavailable due to model uncertainties and, thus, transparency and stability are hard to guarantee.

In order to mitigate the adverse effects of parametric uncertainties in the dynamics of a teleoperation system, different adaptive control methods have been proposed as summarized in the following. Adaptive control schemes for linear master and slave robots have been developed in [14-15]. However, in these papers, an adaptive controller was designed for the slave, but a fixed compensator was used for the master. On the other hand, since robot dynamics are generally nonlinear and multi-degree-of-freedom, adaptive control for nonlinear master and slave models is needed. For this case, adaptive laws were designed for the master and the slave in [16-19]. Moreover, adaptive controllers for nonlinear master and slave dynamics incorporating linear operator and environment dynamics were designed in [20-21].

In terms of the structure of model uncertainties, all teleoperation control related prior art has only considered linearly parameterized (LP) dynamic terms (i.e., terms involving model parameters that appear linearly in the dynamics) but has not considered nonlinearly parameterized (NLP) dynamic terms (i.e., terms involving model 
parameters that appear nonlinearly in the dynamics). Friction, which is ubiquitous in the joints of the master and slave robots of a teleoperation system, is an example of NLP terms. In general, NLP dynamic uncertainties degrade position and force trajectory tracking and, thus, make transparency hard to guarantee if not compensated for in the control scheme.

Outside the realm of teleoperation control, Feemster et al. [22] designed an adaptive controller for NLP dynamic uncertainty compensation. However, the result in [22] can only be applied to setpoint regulation. For trajectory tracking, Hung et al. [23] developed an adaptive controller to compensate for NLP uncertainties in robot manipulators. Nonetheless, the results in [23] have only been applied to motion control of a single robot in free motion. So far, there has been no attempt at simultaneous motion and force control of a master-slave teleoperation system with NLP dynamic uncertainties, in which the master and the slave are allowed to make contact with the human operator and the environment, respectively. The contribution of this paper is in adaptive control of a bilateral teleoperation system encompassing both LP and NLP dynamic uncertainties under both free motion and contact motion conditions. The proposed adaptive controllers for the master and for the slave are then incorporated into the 4-channel bilateral framework to achieve transparent teleoperation.

The organization of this paper is as follows. In Section 2, the nonlinear model of a teleoperation system with NLP dynamic uncertainties is developed. In Section 3, adaptive controllers that can deal with both LP and NLP dynamic uncertainties are designed for the master and for the slave, and incorporated into the 4-channel bilateral teleoperation control framework. Then, transparency of the entire closed-loop system is proved by a Lyapunov function analysis. Examples of system with NLP uncertainties are illustrated in Section 4. In Section 5, numerical simulations are presented comparing the performance of the proposed adaptive controller with that of conventional adaptive controllers. The paper is concluded in Section 6.

\section{Model of a Teleoperation System with NLP Dynamic Terms}

In this section, nonlinear model of a teleoperation system with NLP dynamic uncertainties is developed to facilitate the design of controllers.

\subsection{Dynamics of the master and the slave in joint space}


When interacting with a human operator and an environment, the joint-space dynamic models of $n$-DOF master and the slave robots can be written as

$$
\begin{aligned}
& \mathbf{M}_{m}\left(\mathbf{q}_{m}\right) \ddot{\mathbf{q}}_{m}+\mathbf{C}_{m}\left(\mathbf{q}_{m}, \dot{\mathbf{q}}_{m}\right) \dot{\mathbf{q}}_{m}+\mathbf{G}_{m}\left(\mathbf{q}_{m}\right)-\mathbf{N}_{m}\left(\mathbf{q}_{m}, \dot{\mathbf{q}}_{m}, \boldsymbol{\eta}_{m}\right)=\boldsymbol{\tau}_{m}+\mathbf{J}_{m}^{T} \mathbf{f}_{h} \\
& \mathbf{M}_{s}\left(\mathbf{q}_{s}\right) \ddot{\mathbf{q}}_{s}+\mathbf{C}_{s}\left(\mathbf{q}_{s}, \dot{\mathbf{q}}_{s}\right) \dot{\mathbf{q}}_{s}+\mathbf{G}_{s}\left(\mathbf{q}_{s}\right)-\mathbf{N}_{s}\left(\mathbf{q}_{s}, \dot{\mathbf{q}}_{s}, \boldsymbol{\eta}_{s}\right)=\boldsymbol{\tau}_{s}-\mathbf{J}_{s}^{T} \mathbf{f}_{e}
\end{aligned}
$$

where $\quad \mathbf{q}_{m}$ and $\mathbf{q}_{s} \in \mathfrak{R}^{n \times 1}$ are joint angle positions, $\mathbf{M}_{m}\left(\mathbf{q}_{m}\right)$ and $\mathbf{M}_{s}\left(\mathbf{q}_{s}\right) \in \mathfrak{R}^{n \times n}$ are symmetric positivedefinite inertia matrices, $\mathbf{C}_{m}\left(\mathbf{q}_{m}, \dot{\mathbf{q}}_{m}\right)$ and $\mathbf{C}_{s}\left(\mathbf{q}_{s}, \dot{\mathbf{q}}_{s}\right) \in \mathfrak{R}^{n \times n}$ correspond to Coriolis and centrifugal terms, $\mathbf{G}_{m}\left(\mathbf{q}_{m}\right)$ and $\mathbf{G}_{s}\left(\mathbf{q}_{s}\right) \in \mathfrak{R}^{n \times 1}$ represent gravity terms, $\boldsymbol{\tau}_{m}$ and $\boldsymbol{\tau}_{s} \in \mathfrak{R}^{n \times 1}$ are control torque inputs, and $\mathbf{J}_{m}\left(\mathbf{q}_{m}\right)$ and $\mathbf{J}_{s}\left(\mathbf{q}_{s}\right) \in \mathfrak{R}^{6 \times n} \quad$ are the Jacobian matrices for the master and the slave, respectively. Here, $\mathbf{f}_{h} \in \mathfrak{R}^{6 \times 1}$ is the force that the operator applies to the master and $\mathbf{f}_{e} \in \mathfrak{R}^{6 \times 1}$ is the force that the environment applies to the slave. Also, $\mathbf{N}_{m}\left(\mathbf{q}_{m}, \dot{\mathbf{q}}_{m}, \boldsymbol{\eta}_{m}\right)$ and $\mathbf{N}_{s}\left(\mathbf{q}_{s}, \dot{\mathbf{q}}_{s}, \boldsymbol{\eta}_{s}\right) \in \mathfrak{R}^{n \times 1}$ represent terms whose vectors of parameters $\boldsymbol{\eta}_{m}$ and $\boldsymbol{\eta}_{s}$ are uncertain and appear nonlinearly in the model. In this paper, we assume that the NLP uncertainties in each of the master and slave robots are of a general multiplicative form, i.e.,

$$
N_{i}\left(\mathbf{q}, \dot{\mathbf{q}}, \boldsymbol{\eta}_{i}\right)=R_{i}^{\prime}\left(\mathbf{q}, \dot{\mathbf{q}}, \boldsymbol{\eta}_{i}\right) T_{i}^{\prime}\left(\mathbf{q}, \dot{\mathbf{q}}, \boldsymbol{\eta}_{i}\right)
$$

where $i=1, \ldots, n$ denotes the $i^{t h}$ joint of each robot and $R_{i}^{\prime}\left(\mathbf{q}, \dot{\mathbf{q}}, \boldsymbol{\eta}_{i}\right)$ and $T_{i}^{\prime}\left(\mathbf{q}, \dot{\mathbf{q}}, \boldsymbol{\eta}_{i}\right)$ are nonlinear functions that are assumed to be Lipschitz [24] in $\boldsymbol{\eta}_{i}=\left[\eta_{i 1, . . .}, \eta_{i p_{i}}\right]^{T} \in \mathfrak{R}^{p_{i} \times 1}$. In the following, we will use the subscripts $m$ and $s$ for the master and the slave, respectively.

\subsection{Dynamics of the master and the slave in Cartesian space}

In practice, it is desirable to express the dynamics of the master and the slave robots (and ensure position and force tracking) in the Cartesian space, where the tasks and interactions with the human operator and the environment are naturally specified. Based on (1)-(2), the dynamics of the master and the slave in Cartesian space are

$$
\begin{aligned}
& \mathbf{M}_{x m}\left(\mathbf{q}_{m}\right) \ddot{\mathbf{x}}_{m}+\mathbf{C}_{x m}\left(\mathbf{q}_{m}, \dot{\mathbf{q}}_{m}\right) \dot{\mathbf{x}}_{m}+\mathbf{G}_{x m}\left(\mathbf{q}_{m}\right)-\mathbf{N}_{x m}\left(\mathbf{q}_{m}, \dot{\mathbf{q}}_{m}, \boldsymbol{\eta}_{m}\right)=\mathbf{f}_{m}+\mathbf{f}_{h} \\
& \mathbf{M}_{x s}\left(\mathbf{q}_{s}\right) \ddot{\mathbf{x}}_{s}+\mathbf{C}_{x s}\left(\mathbf{q}_{s}, \dot{\mathbf{q}}_{s}\right) \dot{\mathbf{x}}_{s}+\mathbf{G}_{x S}\left(\mathbf{q}_{s}\right)-\mathbf{N}_{x s}\left(\mathbf{q}_{s}, \dot{\mathbf{q}}_{s}, \boldsymbol{\eta}_{s}\right)=\mathbf{f}_{s}-\mathbf{f}_{e}
\end{aligned}
$$


where, for the corresponding subscripts $m$ and $s$, we have [25]:

$$
\begin{aligned}
& \dot{\mathbf{x}}=\mathbf{J} \dot{\mathbf{q}} \\
& \ddot{\mathbf{x}}=\dot{\mathbf{J}} \dot{\mathbf{q}}+\mathbf{J} \ddot{\mathbf{q}} \\
& \mathbf{M}_{x}(\mathbf{q})=\mathbf{J}^{-T} \mathbf{M}(\mathbf{q}) \mathbf{J}^{-1} \\
& \mathbf{C}_{x}(\mathbf{q}, \dot{\mathbf{q}})=\mathbf{J}^{-T}\left(\mathbf{C}(\mathbf{q}, \dot{\mathbf{q}})-\mathbf{M}(\mathbf{q}) \mathbf{J}^{-1} \mathbf{J}\right) \mathbf{J}^{-1} \\
& \mathbf{G}_{x}(\mathbf{q})=\mathbf{J}^{-T} \mathbf{G}(\mathbf{q}) \\
& \mathbf{N}_{x}(\mathbf{q}, \dot{\mathbf{q}}, \boldsymbol{\eta})=\mathbf{J}^{-T} \mathbf{N}(\mathbf{q}, \dot{\mathbf{q}}, \boldsymbol{\eta}) \\
& \mathbf{f}=\mathbf{J}^{-T} \boldsymbol{\tau}
\end{aligned}
$$

Since the joint-space term $N_{i}\left(\mathbf{q}, \dot{\mathbf{q}}, \boldsymbol{\eta}_{i}\right)$ is of a multiplicative form, the corresponding Cartesian-space term $N_{x i}\left(\mathbf{q}, \dot{\mathbf{q}}, \boldsymbol{\eta}_{i}\right)$ is also of a multiplicative form:

$$
N_{x i}\left(\mathbf{q}, \dot{\mathbf{q}}, \boldsymbol{\eta}_{i}\right)=R_{i}\left(\mathbf{q}, \dot{\mathbf{q}}, \boldsymbol{\eta}_{i}\right) T_{i}\left(\mathbf{q}, \dot{\mathbf{q}}, \boldsymbol{\eta}_{i}\right)
$$

In addition, since $R_{i}^{\prime}\left(\mathbf{q}, \dot{\mathbf{q}}, \boldsymbol{\eta}_{i}\right)$ and $T_{i}^{\prime}\left(\mathbf{q}, \dot{\mathbf{q}}, \boldsymbol{\eta}_{i}\right)$ in (3) are Lipschitz in $\boldsymbol{\eta}_{i}, R_{i}\left(\mathbf{q}, \dot{\mathbf{q}}, \boldsymbol{\eta}_{i}\right)$ and $T_{i}\left(\mathbf{q}, \dot{\mathbf{q}}, \boldsymbol{\eta}_{i}\right)$ in (7) where $R_{i}\left(\mathbf{q}, \dot{\mathbf{q}}, \boldsymbol{\eta}_{i}\right)=\mathbf{J}^{-T} R_{i}^{\prime}\left(\mathbf{q}, \dot{\mathbf{q}}, \boldsymbol{\eta}_{i}\right)$ and $T_{i}\left(\mathbf{q}, \dot{\mathbf{q}}, \boldsymbol{\eta}_{i}\right)=T_{i}^{\prime}\left(\mathbf{q}, \dot{\mathbf{q}}, \boldsymbol{\eta}_{i}\right)$ are also Lipschitz in $\boldsymbol{\eta}_{i}$, i.e.,

$$
\begin{aligned}
& \left\|R_{i}\left(\mathbf{q}, \dot{\mathbf{q}}, \boldsymbol{\eta}_{i}\right)-R_{i}\left(\mathbf{q}, \dot{\mathbf{q}}, \tilde{\boldsymbol{\eta}}_{i}\right)\right\| \leq \sum_{j=1}^{p_{i}} A_{i j}(\mathbf{q}, \dot{\mathbf{q}})\left|\boldsymbol{\eta}_{i j}-\tilde{\boldsymbol{\eta}}_{i j}\right|, \forall \boldsymbol{\eta}_{i}, \tilde{\boldsymbol{\eta}}_{i} \in \boldsymbol{\eta} \\
& \left\|T_{i}\left(\mathbf{q}, \dot{\mathbf{q}}, \boldsymbol{\eta}_{i}\right)-T_{i}\left(\mathbf{q}, \dot{\mathbf{q}}, \tilde{\boldsymbol{\eta}}_{i}\right)\right\| \leq \sum_{j=1}^{p_{i}} Z_{i j}(\mathbf{q}, \dot{\mathbf{q}})\left|\boldsymbol{\eta}_{i j}-\tilde{\boldsymbol{\eta}}_{i j}\right|, \forall \boldsymbol{\eta}_{i}, \tilde{\boldsymbol{\eta}}_{i} \in \boldsymbol{\eta}
\end{aligned}
$$

In the above, $p_{i}$ is the dimension of $\boldsymbol{\eta}_{i}$ in (3). Also, $A_{i j}(\mathbf{q}, \dot{\mathbf{q}}), Z_{i j}(\mathbf{q}, \dot{\mathbf{q}}) \geq 0$ are continuous functions.

Property 1 [26]. The first three terms in the left sides of (4) and (5) are linear in a set of parameters $\boldsymbol{\alpha}=\left[\alpha_{1}, \ldots, \alpha_{r}\right]^{T}$ such that

$$
\mathbf{M}_{x}(\mathbf{q}) \ddot{\mathbf{x}}+\mathbf{C}_{X}(\mathbf{q}, \dot{\mathbf{q}}) \dot{\mathbf{x}}+\mathbf{G}_{x}(\mathbf{q})=\mathbf{Y}(\mathbf{q}, \dot{\mathbf{q}}, \dot{\mathbf{x}}, \ddot{\mathbf{x}}) \boldsymbol{\alpha}
$$

where $\mathbf{Y} \in \mathfrak{R}^{n \times r}$ is a regressor matrix.

Property 2 [26]. The matrix $\dot{\mathbf{M}}_{x}(\mathbf{q})-2 \mathbf{C}_{x}(\mathbf{q}, \dot{\mathbf{q}})$ is skew-symmetric:

$$
\zeta^{T}\left(\dot{\mathbf{M}}_{x}(\mathbf{q})-2 \mathbf{C}_{x}(\mathbf{q}, \dot{\mathbf{q}})\right) \xi=0, \forall \xi \in \Re^{n \times 1}
$$

Property 3 [23]. For $\forall e(t) \in \Re$, the Lipschitz functions $R_{i}\left(\mathbf{q}, \dot{\mathbf{q}}, \boldsymbol{\eta}_{i}\right)$ and $T_{i}\left(\mathbf{q}, \dot{\mathbf{q}}, \boldsymbol{\eta}_{i}\right)$ satisfy the following inequality: 


$$
\begin{aligned}
& e(t) R_{i}\left(\mathbf{q}, \dot{\mathbf{q}}, \boldsymbol{\eta}_{i}\right) T_{i}\left(\mathbf{q}, \dot{\mathbf{q}}, \boldsymbol{\eta}_{i}\right) \\
& \leq e(t) R_{i}(\mathbf{q}, \dot{\mathbf{q}}, 0) T_{i}(\mathbf{q}, \dot{\mathbf{q}}, 0)+|e(t)| \\
& \left\{A_{i}(\mathbf{q}, \dot{\mathbf{q}}) Z_{i}(\mathbf{q}, \dot{\mathbf{q}})\left(\sum_{j=1}^{p_{i}} \eta_{i j}\right)^{2}+\left[\left\|T_{i}(\mathbf{q}, \dot{\mathbf{q}}, 0)\right\| A_{i}(\mathbf{q}, \dot{\mathbf{q}})+\left\|R_{i}(\mathbf{q}, \dot{\mathbf{q}}, 0)\right\| Z_{i}(\mathbf{q}, \dot{\mathbf{q}})\right] \sum_{j=1}^{p_{i}} \eta_{i j}\right\}
\end{aligned}
$$

where $A_{i}(\mathbf{q}, \dot{\mathbf{q}}):=\max _{j=1,2, \ldots, p_{i}} A_{i j}(\mathbf{q}, \dot{\mathbf{q}})$ and $Z_{i}(\mathbf{q}, \dot{\mathbf{q}}):=\max _{j=1,2, \ldots, p_{i}} Z_{i j}(\mathbf{q}, \dot{\mathbf{q}})$

Lemma 1. The NLP terms $\mathbf{N}_{x}(\mathbf{q}, \dot{\mathbf{q}}, \boldsymbol{\eta})$ in (4) and (5) satisfy

$$
\mathbf{s}^{T} \mathbf{N}_{x}(\mathbf{q}, \dot{\mathbf{q}}, \boldsymbol{\eta}) \leq \mathbf{s}^{T} \mathbf{N}_{x}(\mathbf{q}, \dot{\mathbf{q}}, \mathbf{0})+\mathbf{s}^{T} \mathbf{Q}(\mathbf{s}, \mathbf{q}, \dot{\mathbf{q}}) \boldsymbol{\theta}
$$

where $\mathbf{s}=\Delta \dot{\mathbf{x}}+\lambda \Delta \mathbf{x} \in \mathfrak{R}^{1 \times n}, \Delta \mathbf{x}=\mathbf{x}-\mathbf{x}_{d}$ is the position error between the actual position $\mathbf{x}$ and the desired position $\mathbf{x}_{d}, \Delta \dot{\mathbf{x}}$ is the velocity error, $\lambda$ is a diagonal positive definite matrix, and

$$
\begin{gathered}
\mathbf{Q}(\mathbf{s}, \mathbf{q}, \dot{\mathbf{q}}):=\operatorname{diag}\left[\operatorname{sgn}\left(s_{1}\right) \mathbf{w}_{1}(\mathbf{q}, \dot{\mathbf{q}}), \ldots, \operatorname{sgn}\left(s_{n}\right) \mathbf{w}_{n}(\mathbf{q}, \dot{\mathbf{q}})\right] \in \mathfrak{R}^{n \times 2 n} \\
\mathbf{w}_{i}:=\left[A_{i}(\mathbf{q}, \dot{\mathbf{q}}) Z_{i}(\mathbf{q}, \dot{\mathbf{q}}) \quad\left\|T_{i}(\mathbf{q}, \dot{\mathbf{q}}, \mathbf{0})\right\| A_{i}(\mathbf{q}, \dot{\mathbf{q}})+\left\|R_{i}(\mathbf{q}, \dot{\mathbf{q}}, \mathbf{0})\right\| Z_{i}(\mathbf{q}, \dot{\mathbf{q}})\right] \in \mathfrak{R}^{1 \times 2} \\
\boldsymbol{\theta}:=\left[\begin{array}{llll}
\boldsymbol{\theta}_{1}^{T} & \boldsymbol{\theta}_{2}^{T} & \ldots & \boldsymbol{\theta}_{n}^{T}
\end{array}\right]^{T} \in \mathfrak{R}^{2 n \times 1} \\
\boldsymbol{\theta}_{i}:=\left[\begin{array}{lll}
\left(\sum_{j=1}^{p_{i}} \eta_{i j}\right)^{2} & \sum_{j=1}^{p_{i}} \eta_{i j}
\end{array}\right]^{T} .
\end{gathered}
$$

Proof: According to the definitions of $\mathbf{s}$ and $\mathbf{N}_{x}(\mathbf{q}, \dot{\mathbf{q}}, \boldsymbol{\eta})$, we have $\mathbf{s}^{T} \mathbf{N}_{x}(\mathbf{q}, \dot{\mathbf{q}}, \boldsymbol{\eta})=\sum_{i=1}^{n} s_{i} N_{x i}\left(\mathbf{q}, \dot{\mathbf{q}}, \boldsymbol{\eta}_{i}\right)$. Using Property 3, we obtain

$$
\begin{aligned}
& \sum_{i=1}^{n} s_{i} N_{x i}\left(\mathbf{q}, \dot{\mathbf{q}}, \boldsymbol{\eta}_{i}\right) \\
\leq & \sum_{i=1}^{n} s_{i} R_{i}(\mathbf{q}, \dot{\mathbf{q}}, 0) T_{i}(\mathbf{q}, \dot{\mathbf{q}}, 0)+\sum_{i=1}^{n}\left|s_{i}\right| \\
& \left\{A_{i}(\mathbf{q}, \dot{\mathbf{q}}) Z_{i}(\mathbf{q}, \dot{\mathbf{q}})\left(\sum_{j=1}^{p_{i}} \eta_{i j}\right)^{2}+\left[\left\|T_{i}(\mathbf{q}, \dot{\mathbf{q}}, 0)\right\| A_{i}(\mathbf{q}, \dot{\mathbf{q}})+\left\|R_{i}(\mathbf{q}, \dot{\mathbf{q}}, 0)\right\| Z_{i}(\mathbf{q}, \dot{\mathbf{q}})\right] \sum_{j=1}^{p_{i}} \eta_{i j}\right\}
\end{aligned}
$$

Using the definitions of $\mathbf{Q}(\mathbf{s}, \mathbf{q}, \dot{\mathbf{q}})$ and $\boldsymbol{\theta}$, and $\left|s_{i}\right|=s_{i} \operatorname{sgn}\left(s_{i}\right)$, we get

$$
\mathbf{s}^{T} \mathbf{N}_{\chi}(\mathbf{q}, \dot{\mathbf{q}}, \boldsymbol{\eta}) \leq \mathbf{s}^{T} \mathbf{N}_{\chi}(\mathbf{q}, \dot{\mathbf{q}}, \mathbf{0})+\mathbf{s}^{T} \mathbf{Q}(\mathbf{s}, \mathbf{q}, \dot{\mathbf{q}}) \boldsymbol{\theta}
$$




\section{Adaptive Bilateral Teleoperation Control}

Due to model uncertainties, perfect knowledge of the master and slave dynamics may not be available. When the master and the slave experience parametric uncertainties in their dynamics, according to Property 1 ,

$$
\begin{aligned}
& \hat{\mathbf{M}}_{x m}\left(\mathbf{q}_{m}\right) \ddot{\mathbf{x}}_{m}+\hat{\mathbf{C}}_{x m}\left(\mathbf{q}_{m}, \dot{\mathbf{q}}_{m}\right) \dot{\mathbf{x}}_{m}+\hat{\mathbf{G}}_{x m}\left(\mathbf{q}_{m}\right)=\mathbf{Y}_{m}\left(\mathbf{q}_{m}, \dot{\mathbf{q}}_{m}, \dot{\mathbf{x}}_{m}, \ddot{\mathbf{x}}_{m}\right) \hat{\boldsymbol{\alpha}}_{m} \\
& \hat{\mathbf{M}}_{x s}\left(\mathbf{q}_{s}\right) \ddot{\mathbf{x}}_{s}+\hat{\mathbf{C}}_{x s}\left(\mathbf{q}_{s}, \dot{\mathbf{q}}_{s}\right) \dot{\mathbf{x}}_{s}+\hat{\mathbf{G}}_{x s}\left(\mathbf{q}_{s}\right)=\mathbf{Y}_{s}\left(\mathbf{q}_{s}, \dot{\mathbf{q}}_{s}, \dot{\mathbf{x}}_{s}, \ddot{\mathbf{x}}_{s}\right) \hat{\boldsymbol{\alpha}}_{s}
\end{aligned}
$$

where $\hat{\boldsymbol{\alpha}}_{m}$ and $\hat{\boldsymbol{\alpha}}_{s}$ are estimates of the dynamics parameter vectors $\boldsymbol{\alpha}_{m}$ and $\boldsymbol{\alpha}_{s}$, respectively. Furthermore, parameter vectors $\boldsymbol{\eta}_{m}$ and $\boldsymbol{\eta}_{s}$ in the terms $\mathbf{N}_{x m}\left(\mathbf{q}_{m}, \dot{\mathbf{q}}_{m}, \boldsymbol{\eta}_{m}\right)$ and $\mathbf{N}_{x s}\left(\mathbf{q}_{s}, \dot{\mathbf{q}}_{s}, \boldsymbol{\eta}_{s}\right)$ in (4) and (5) are not perfectly known. In such a case, some parameters of $\boldsymbol{\eta}_{m}$ and $\boldsymbol{\eta}_{s}$ appear nonlinearly in $\mathbf{N}_{x m}\left(\mathbf{q}_{m}, \dot{\mathbf{q}}_{m}, \boldsymbol{\eta}_{m}\right)$ and $\mathbf{N}_{x S}\left(\mathbf{q}_{s}, \dot{\mathbf{q}}_{s}, \boldsymbol{\eta}_{s}\right)$, respectively. Our goal is to design a control scheme for a teleoperation system with both LP and NLP dynamic uncertainties (i.e., uncertainties both in $\boldsymbol{\alpha}_{m}$ and $\boldsymbol{\alpha}_{s}$ and in $\boldsymbol{\eta}_{m}$ and $\boldsymbol{\eta}_{m}$ ) such that transparency is guaranteed.

\subsection{Architecture of the proposed teleoperation controller}

In an ideally transparent teleoperation system, through appropriate control signals, the master and the slave positions and forces will match regardless of the operator and environment dynamics, i.e., $\mathbf{x}_{m}=\mathbf{x}_{s}, \mathbf{f}_{h}=\mathbf{f}_{e}$. For achieving this ideal response, various teleoperation control architectures have been proposed. These control architectures are usually classified as position error based (PEB), direct force reflection (DFR), shared compliance control (SCC), and 4-channel (4CH) control methods [27]. Among them, the 4-channel architecture is the most general case and one that can achieve perfect transparency. As seen in Fig. 2, the 4channel architecture has a position controller comprised of the blocks $\mathbf{C}_{L}$ and $\mathbf{C}_{4}$ for the master, and another position controller comprised of $\mathbf{C}_{R}$ and $\mathbf{C}_{1}$ for the slave. The controllers $\mathbf{C}_{2}$ and $\mathbf{C}_{3}$ are feedforward force terms for the master and the slave, respectively. Also, $\mathbf{C}_{5}$ and $\mathbf{C}_{6}$ are local force feedback controllers for the master and for the slave, respectively. The signals $\mathbf{f}_{h}^{*}$ and $\mathbf{f}_{e}^{*}$ denote the exogenous forces of the operator and 
the environment, respectively. The 4-channel architecture can be reduced to the PEB, DFR, or SCC architectures through proper choices of $\mathbf{C}_{L}, \mathbf{C}_{R}$, and $\mathbf{C}_{1}$ to $\mathbf{C}_{6}$.

The basic idea of our proposed teleoperation control scheme involves incorporating two adaptive position controllers for the master and the slave into the 4-channel teleoperation architecture. To this end, in Fig. 2, blocks $\mathbf{C}_{L}$ and $\mathbf{C}_{4}$ are replaced by an adaptive position controller for the master, and blocks $\mathbf{C}_{R}$ and $\mathbf{C}_{1}$ are replaced by another adaptive position controller for the slave (see the dashed boxes in Fig. 2). Blocks $\mathbf{C}_{2}, \mathbf{C}_{3}$, $\mathbf{C}_{5}$ and $\mathbf{C}_{6}$ are still maintained as force feedforward and feedback controllers in the proposed approach, where the choices $\mathbf{C}_{2}=\mathbf{I}+\mathbf{C}_{6}$ and $\mathbf{C}_{3}=\mathbf{I}+\mathbf{C}_{5}$ are made for transparency reasons ( $\mathbf{I}$ is the identity matrix).

\subsection{Design of control laws and adaptation laws}

With the architecture in Section 3.1, we now begin to design the adaptive controllers for the master and the slave. According to the definition of $\mathbf{s}$ in Lemma 1, define the following two vectors $\mathbf{s}_{m}, \mathbf{s}_{s} \in \mathfrak{R}^{n}$ for the master and for the slave, respectively:

$$
\begin{aligned}
& \mathbf{s}_{m}=\Delta \dot{\mathbf{x}}_{m}+\lambda \Delta \mathbf{x}_{m}=\dot{\mathbf{x}}_{m}-\dot{\mathbf{x}}_{m r} \\
& \mathbf{s}_{s}=\Delta \dot{\mathbf{x}}_{s}+\lambda \Delta \mathbf{x}_{s}=\dot{\mathbf{x}}_{s}-\dot{\mathbf{x}}_{s r}
\end{aligned}
$$

where, as mentioned before, $\Delta \mathbf{x}_{m}=\mathbf{x}_{m}-\mathbf{x}_{s}, \Delta \mathbf{x}_{s}=\mathbf{x}_{s}-\mathbf{x}_{m}, \dot{\mathbf{x}}_{m r}=\dot{\mathbf{x}}_{s}-\lambda \Delta \mathbf{x}_{m}, \dot{\mathbf{x}}_{s r}=\dot{\mathbf{x}}_{m}-\lambda \Delta \mathbf{x}_{s}$, and $\lambda$ is a diagonal positive definite matrix. From (11)-(12), we get

$$
\begin{aligned}
& \dot{\mathbf{x}}_{m}=\mathbf{s}_{m}+\dot{\mathbf{x}}_{m r}, \quad \dot{\mathbf{x}}_{s}=\mathbf{s}_{s}+\dot{\mathbf{x}}_{s r} \\
& \ddot{\mathbf{x}}_{m}=\dot{\mathbf{s}}_{m}+\ddot{\mathbf{x}}_{m r}, \quad \ddot{\mathbf{x}}_{s}=\dot{\mathbf{s}}_{s}+\ddot{\mathbf{x}}_{s r}
\end{aligned}
$$

Substituting (13)-(14) into (4)-(5), the open-loop dynamics of the master and the slave become

$$
\begin{aligned}
& \mathbf{M}_{x m}\left(\mathbf{q}_{m}\right) \dot{\mathbf{s}}_{m}+\mathbf{C}_{x m}\left(\mathbf{q}_{m}, \dot{\mathbf{q}}_{m}\right) \mathbf{s}_{m}=\mathbf{f}_{m}+\mathbf{f}_{h}-\mathbf{Y}_{m r}\left(\mathbf{q}_{m}, \dot{\mathbf{q}}_{m}, \dot{\mathbf{x}}_{m r}, \ddot{\mathbf{x}}_{m r}\right) \boldsymbol{\alpha}_{m}+\mathbf{N}_{x m}\left(\mathbf{q}_{m}, \dot{\mathbf{q}}_{m}, \mathbf{\eta}_{m}\right) \\
& \mathbf{M}_{x s}\left(\mathbf{q}_{s}\right) \dot{\mathbf{s}}_{s}+\mathbf{C}_{x s}\left(\mathbf{q}_{s}, \dot{\mathbf{q}}_{s}\right) \mathbf{s}_{s}=\mathbf{f}_{s}-\mathbf{f}_{e}-\mathbf{Y}_{s r}\left(\mathbf{q}_{s}, \dot{\mathbf{q}}_{s}, \dot{\mathbf{x}}_{s r}, \ddot{\mathbf{x}}_{s r}\right) \boldsymbol{\alpha}_{s}+\mathbf{N}_{x s}\left(\mathbf{q}_{s}, \dot{\mathbf{q}}_{s}, \mathbf{\eta}_{s}\right)
\end{aligned}
$$

where

$$
\begin{aligned}
& \mathbf{Y}_{m r}\left(\mathbf{q}_{m}, \dot{\mathbf{q}}_{m}, \dot{\mathbf{x}}_{m r}, \ddot{\mathbf{x}}_{m r}\right) \boldsymbol{\alpha}_{m}=\mathbf{M}_{x m}\left(\mathbf{q}_{m}\right) \ddot{\mathbf{x}}_{m r}+\mathbf{C}_{x m}\left(\mathbf{q}_{m}, \dot{\mathbf{q}}_{m}\right) \dot{\mathbf{x}}_{m r}+\mathbf{G}_{x m}\left(\mathbf{q}_{m}\right) \\
& \mathbf{Y}_{s r}\left(\mathbf{q}_{s}, \dot{\mathbf{q}}_{s}, \dot{\mathbf{x}}_{s r}, \ddot{\mathbf{x}}_{s r}\right) \boldsymbol{\alpha}_{s}=\mathbf{M}_{x s}\left(\mathbf{q}_{s}\right) \ddot{\mathbf{x}}_{s r}+\mathbf{C}_{x s}\left(\mathbf{q}_{s}, \dot{\mathbf{q}}_{s}\right) \dot{\mathbf{x}}_{s r}+\mathbf{G}_{x s}\left(\mathbf{q}_{s}\right)
\end{aligned}
$$


Now, the control laws and the adaptation laws for the master and the slave are proposed as the following:

- Control laws:

$$
\begin{aligned}
& \mathbf{f}_{m}=-\mathbf{K}_{m} \mathbf{s}_{m}+\mathbf{Y}_{m r} \hat{\boldsymbol{\alpha}}_{m}-\mathbf{N}_{x m}\left(\mathbf{q}_{m}, \dot{\mathbf{q}}_{m}, \mathbf{0}\right)-\mathbf{Q}_{m} \hat{\boldsymbol{\theta}}_{m}+\mathbf{C}_{2}\left(\mathbf{f}_{h}-\mathbf{f}_{e}\right)-\mathbf{f}_{h} \\
& \mathbf{f}_{s}=-\mathbf{K}_{s} \mathbf{s}_{s}+\mathbf{Y}_{s r} \hat{\boldsymbol{\alpha}}_{s}-\mathbf{N}_{x s}\left(\mathbf{q}_{s}, \dot{\mathbf{q}}_{s}, \mathbf{0}\right)-\mathbf{Q}_{s} \hat{\boldsymbol{\theta}}_{s}+\mathbf{C}_{3}\left(\mathbf{f}_{h}-\mathbf{f}_{e}\right)+\mathbf{f}_{e}
\end{aligned}
$$

where $\mathbf{K}_{m}, \mathbf{K}_{s}, \mathbf{C}_{2}$ and $\mathbf{C}_{3}$ are all diagonal positive-definite matrices (or positive constants), and $\hat{\boldsymbol{\alpha}}_{m}, \hat{\boldsymbol{\theta}}_{m}$, $\hat{\boldsymbol{\alpha}}_{s}$, and $\hat{\boldsymbol{\theta}}_{s}$ are estimates of $\boldsymbol{\alpha}_{m}, \boldsymbol{\theta}_{m}, \boldsymbol{\alpha}_{s}$, and $\boldsymbol{\theta}_{s}$, respectively. Note that $\mathbf{Q}_{m}, \mathbf{Q}_{s}, \boldsymbol{\theta}_{m}$ and $\boldsymbol{\theta}_{s}$ have been introduced in Lemma 1.

- Adaptation laws for the LP uncertain parameters in dynamics:

$$
\dot{\hat{\boldsymbol{\alpha}}}_{m}=-\boldsymbol{\Gamma}_{a m} \mathbf{Y}_{m r}^{T} \mathbf{s}_{m}, \dot{\hat{\boldsymbol{\alpha}}}_{s}=-\boldsymbol{\Gamma}_{a s} \mathbf{Y}_{s r}^{T} \mathbf{s}_{s}
$$

where $\boldsymbol{\Gamma}_{\alpha m}$ and $\boldsymbol{\Gamma}_{\alpha s}$ are constant positive-definite matrices.

- Adaptation laws for the NLP uncertain parameters in dynamics:

$$
\dot{\hat{\boldsymbol{\theta}}}_{m}=\boldsymbol{\Gamma}_{\theta m} \mathbf{Q}_{m}^{T} \mathbf{s}_{m}, \dot{\hat{\boldsymbol{\theta}}}_{s}=\boldsymbol{\Gamma}_{\theta s} \mathbf{Q}_{s}^{T} \mathbf{s}_{s}
$$

where $\boldsymbol{\Gamma}_{\theta m}$ and $\boldsymbol{\Gamma}_{\theta s}$ are constant positive-definite matrices.

Each of the control laws (19)-(20) includes six terms. The first term is a feedback law involving the velocity and position tracking errors between the master and the slave, the second term compensates for the LP dynamic uncertainties, and the third and the fourth terms compensate for the NLP dynamic uncertainties. These first four terms together perform adaptive position control as shown in Fig. 2. The fifth term implements force tracking between the master and the slave (i.e., force feedback to the operator), and the sixth term cancels the human/master and the slave/environment interaction force in the master and the slave closed-loop dynamics, respectively. The LP uncertain parameter vectors $\boldsymbol{\alpha}_{m}, \boldsymbol{\alpha}_{s}$ are updated by (21) and the NLP uncertain parameter vectors $\boldsymbol{\theta}_{m}, \boldsymbol{\theta}_{s}$ are updated by (22).

Substituting the control laws (19)-(20) into the open-loop dynamics (15)-(16), the closed-loops dynamics for the master and the slave are obtained as

$$
\begin{aligned}
\mathbf{M}_{x m}\left(\mathbf{q}_{m}\right) \dot{\mathbf{s}}_{m}+\mathbf{C}_{x m}\left(\mathbf{q}_{m}, \dot{\mathbf{q}}_{m}\right) \mathbf{s}_{m}= & -\mathbf{K}_{m} \mathbf{s}_{m}+\mathbf{Y}_{m r} \Delta \boldsymbol{\alpha}_{m} \\
& +\mathbf{N}_{x m}\left(\mathbf{q}_{m}, \dot{\mathbf{q}}_{m}, \boldsymbol{\eta}_{m}\right)-\mathbf{N}_{x m}\left(\mathbf{q}_{m}, \dot{\mathbf{q}}_{m}, \mathbf{0}\right)-\mathbf{Q}_{m} \hat{\boldsymbol{\theta}}_{m}+\mathbf{C}_{2}\left(\mathbf{f}_{h}-\mathbf{f}_{e}\right)
\end{aligned}
$$




$$
\begin{aligned}
\mathbf{M}_{x S}\left(\mathbf{q}_{s}\right) \dot{\mathbf{s}}_{s}+\mathbf{C}_{x s}\left(\mathbf{q}_{s}, \dot{\mathbf{q}}_{s}\right) \mathbf{s}_{s}= & -\mathbf{K}_{s} \mathbf{s}_{s}+\mathbf{Y}_{s r} \Delta \boldsymbol{\alpha}_{s} \\
& +\mathbf{N}_{x s}\left(\mathbf{q}_{s}, \dot{\mathbf{q}}_{s}, \boldsymbol{\eta}_{s}\right)-\mathbf{N}_{x s}\left(\mathbf{q}_{s}, \dot{\mathbf{q}}_{s}, \mathbf{0}\right)-\mathbf{Q}_{s} \hat{\boldsymbol{\theta}}_{s}+\mathbf{C}_{3}\left(\mathbf{f}_{h}-\mathbf{f}_{e}\right)
\end{aligned}
$$

where $\Delta \boldsymbol{\alpha}_{m}=\hat{\boldsymbol{\alpha}}_{m}-\boldsymbol{\alpha}_{m}$ and $\Delta \boldsymbol{\alpha}_{s}=\hat{\boldsymbol{\alpha}}_{s}-\boldsymbol{\alpha}_{s}$. Multiplying both sides of (24) by $\mathbf{C}_{2} \mathbf{C}_{3}^{-1}$, subtracting the result from (23), and using $\mathbf{s}_{s}=-\mathbf{s}_{m}$ gives us a unified closed-loop equation for the entire master-slave system as

$$
\begin{aligned}
& \left(\mathbf{M}_{x m}\left(\mathbf{q}_{m}\right)+\mathbf{C}_{2} \mathbf{C}_{3}{ }^{-1} \mathbf{M}_{x s}\left(\mathbf{q}_{s}\right)\right) \dot{\mathbf{s}}_{m}+\left(\mathbf{C}_{x m}\left(\mathbf{q}_{m}, \dot{\mathbf{q}}_{m}\right)+\mathbf{C}_{2} \mathbf{C}_{3}^{-1} \mathbf{C}_{x s}\left(\mathbf{q}_{s}\right)\right) \mathbf{s}_{m} \\
= & -\left(\mathbf{K}_{m}+\mathbf{C}_{2} \mathbf{C}_{3}{ }^{-1} \mathbf{K}_{s}\right) \mathbf{s}_{m}+\mathbf{Y}_{m r} \Delta \boldsymbol{\alpha}_{m}+\mathbf{N}_{x m}\left(\mathbf{q}_{m}, \dot{\mathbf{q}}_{m}, \boldsymbol{\eta}_{m}\right)-\mathbf{N}_{x m}\left(\mathbf{q}_{m}, \dot{\mathbf{q}}_{m}, \mathbf{0}\right)-\mathbf{Q}_{m} \hat{\boldsymbol{\theta}}_{m} \\
& -\mathbf{C}_{2} \mathbf{C}_{3}{ }^{-1} \mathbf{Y}_{s r} \Delta \boldsymbol{\alpha}_{s}-\mathbf{C}_{2} \mathbf{C}_{3}{ }^{-1} \mathbf{N}_{x s}\left(\mathbf{q}_{s}, \dot{\mathbf{q}}_{s}, \mathbf{\eta}_{s}\right)+\mathbf{C}_{2} \mathbf{C}_{3}{ }^{-1} \mathbf{N}_{x s}\left(\mathbf{q}_{s}, \dot{\mathbf{q}}_{s}, \mathbf{0}\right)+\mathbf{C}_{2} \mathbf{C}_{3}{ }^{-1} \mathbf{Q}_{s} \hat{\boldsymbol{\theta}}_{s}
\end{aligned}
$$

Remark 1: In (1)-(2), the term $\mathbf{N}(\mathbf{q}, \dot{\mathbf{q}}, \boldsymbol{\eta})$ involves NLP uncertainties. If $\mathbf{N}(\mathbf{q}, \dot{\mathbf{q}}, \boldsymbol{\eta})$ contains both LP and NLP uncertainties, the LP parameters can be incorporated into $\boldsymbol{\alpha}_{m}$ and $\boldsymbol{\alpha}_{s}$ and updated in (21), while the NLP parameters enter into $\hat{\boldsymbol{\theta}}_{m}$ and $\hat{\boldsymbol{\theta}}_{s}$ and are updated in (22).

Remark 2: In the controllers (19)-(22), switching activities may exist because of the discontinuous function sgn() in $\mathbf{Q}_{m}$ and $\mathbf{Q}_{s}$, which may be undesirable. This can be resolved by replacing $\operatorname{sgn}()$ with a smooth saturation function, e.g.,

$$
s_{i}=\left\{\begin{array}{lll}
1 & \text { if } & s_{i}>\varepsilon \\
-1 & \text { if } & s_{i}<\varepsilon \\
\frac{s_{i}}{\varepsilon} & \text { if } & -\varepsilon \leq s_{i} \leq \varepsilon
\end{array}\right.
$$

where $i=1, \ldots, n$, and $\varepsilon$ is a small positive constant.

Remark 3: In the adaptive control laws (19)-(20), the interaction force between the master and the operator $\mathbf{f}_{h}$ and the interaction force between the slave and the environment $\mathbf{f}_{e}$ are assumed to be measured by force sensors and are included in order to cancel the same terms in the master and the slave dynamics (4)-(5). Alternatively, $\mathbf{f}_{h}$ and $\mathbf{f}_{e}$ in (4)-(5) can be replaced by the following dynamic models for the human operator's hand and the environment, which have successfully been used by other researchers [20-21]:

$$
\begin{aligned}
& \mathbf{f}_{h}=\mathbf{f}_{h}^{*}-\left(\mathbf{M}_{h} \ddot{\mathbf{x}}_{m}+\mathbf{B}_{\mathbf{h}} \dot{\mathbf{x}}_{m}+\mathbf{K}_{h} \mathbf{x}_{m}\right) \\
& \mathbf{f}_{e}=\mathbf{M}_{e} \ddot{\mathbf{x}}_{s}+\mathbf{B}_{e} \dot{\mathbf{x}}_{s}+\mathbf{K}_{e} \mathbf{x}_{s}
\end{aligned}
$$


where $\mathbf{M}_{h}, \mathbf{B}_{h}, \mathbf{K}_{h}, \mathbf{M}_{e}, \mathbf{B}_{e}$ and $\mathbf{K}_{e}$ are symmetric positive-definite constant matrices corresponding to the mass, damping, and stiffness of the operator's hand and the environment, respectively. After substituting (26)(27) into (4)-(5), combined models for the master/operator system and the slave/environment system will be obtained as follows:

$$
\begin{aligned}
& \left(\mathbf{M}_{x m}\left(\mathbf{q}_{m}\right)+\mathbf{M}_{h}\right) \ddot{\mathbf{x}}_{m}+\left(\mathbf{C}_{x m}\left(\mathbf{q}_{m}, \dot{\mathbf{q}}_{m}\right)+\mathbf{B}_{h}\right) \dot{\mathbf{x}}_{m}+\mathbf{K}_{h} \mathbf{x}_{m}+\mathbf{G}_{x m}\left(\mathbf{q}_{m}\right)-\mathbf{f}_{h}^{*}-\mathbf{N}_{x m}\left(\mathbf{q}_{m}, \dot{\mathbf{q}}_{m}, \mathbf{\eta}_{m}\right)=\mathbf{f}_{m} \\
& \left(\mathbf{M}_{x s}\left(\mathbf{q}_{s}\right)+\mathbf{M}_{e}\right) \ddot{\mathbf{x}}_{s}+\left(\mathbf{C}_{x s}\left(\mathbf{q}_{s}, \dot{\mathbf{q}}_{s}\right)+\mathbf{B}_{e}\right) \dot{\mathbf{x}}_{s}+\mathbf{K}_{e} \mathbf{x}_{s}+\mathbf{G}_{x s}\left(\mathbf{q}_{s}\right)-\mathbf{N}_{x s}\left(\mathbf{q}_{s}, \dot{\mathbf{q}}_{s}, \boldsymbol{\eta}_{s}\right)=\mathbf{f}_{s}
\end{aligned}
$$

For the combined models (28)-(29), new adaptive controllers can be designed in a fashion similar to (19)-(22) but without the last terms $\mathbf{f}_{h}$ and $\mathbf{f}_{e}$ in the control laws (19)-(20) as they have already been incorporated into the dynamic models of the master and the slave. In this case, all dynamics uncertainties in the operator, the master, the slave, and the environment are encompassed in the models and need to be adapted to.

\subsection{Transparency of the closed-loop teleoperation system}

Let us go back to the original design where we found the unified closed-loop equation (25) for the overall teleoperation system based on the master closed-loop dynamics (23) and the slave closed-loop dynamics (24). Thus, we can use a unified Lyapunov function to show the transparency of the overall system in the following theorem.

Lemma 2: If $\boldsymbol{\varphi}(t), \dot{\varphi}(t) \in L_{\infty}$ (i.e., $\boldsymbol{\varphi}(t)$ and $\dot{\boldsymbol{\varphi}}(t)$ are bounded), and if $\boldsymbol{\varphi}(t) \in L_{2}$ (i.e., $\boldsymbol{\varphi}(t)$ is square integrable), then $\lim _{t \rightarrow \infty} \varphi(t) \rightarrow 0$

Proof : Let $\mathbf{f}(t)=\boldsymbol{\varphi}^{2}(t)$, then $\dot{\mathbf{f}}(t)=2 \boldsymbol{\varphi}(t) \dot{\boldsymbol{\varphi}}(t)$. Therefore, $\dot{\mathbf{f}}(t)$ is bounded since both $\boldsymbol{\varphi}(t)$ and $\dot{\boldsymbol{\varphi}}(t)$ are bounded. Thus, $\mathbf{f}(t)$ is uniformly continuous. On the other hand, since $\boldsymbol{\varphi}(t) \in L_{2}$ (i.e., $\int_{0}^{\infty} \boldsymbol{\varphi}^{2}(t) d t<\infty$ ), we get $\int_{0}^{\infty} \mathbf{f}(t) d t<\infty$. Using Barbalat's Lemma [26], we have $\lim _{t \rightarrow \infty} \mathbf{f}(t)=0$ and hence $\lim _{t \rightarrow \infty} \varphi(t)=0$.

Theorem 1: Consider that the nonlinear teleoperation system (4)-(5) has both LP and NLP dynamic uncertainties and is controlled by the adaptive control laws (19)-(20) using the LP dynamic adaptation laws (21) and the NLP dynamic adaptation laws (22). Also, assume the following conditions holds: 
1) The Jacobian matrices $\mathbf{J}_{m}$ and $\mathbf{J}_{s}$ are nonsingular,

2) The force control gain matrices $\mathbf{C}_{2}$ and $\mathbf{C}_{3}$ are invertible.

Then, the signals $\mathbf{s}_{m}, \Delta \boldsymbol{\alpha}_{m}, \Delta \boldsymbol{\theta}_{m}, \Delta \boldsymbol{\alpha}_{s}, \Delta \boldsymbol{\theta}_{s}$ are bounded. Moreover, the position tracking error $\Delta \mathbf{x}_{m}=\mathbf{x}_{m}-\mathbf{x}_{s}$ converges to zero as $t \rightarrow \infty$, and the force tracking error $\Delta \mathbf{f}=\mathbf{f}_{h}-\mathbf{f}_{e}$ remains bounded.

Proof: Consider the Lyapunov function candidate

$$
\begin{aligned}
V= & \frac{1}{2} \mathbf{s}_{m}^{T}\left(\mathbf{M}_{x m}\left(\mathbf{q}_{m}\right)+\mathbf{C}_{2} \mathbf{C}_{3}^{-1} \mathbf{M}_{x s}\left(\mathbf{q}_{s}\right)\right) \mathbf{s}_{m} \\
& +\frac{1}{2} \Delta \boldsymbol{\alpha}_{m}^{T} \boldsymbol{\Gamma}_{\alpha m}^{-1} \Delta \boldsymbol{\alpha}_{m}+\frac{1}{2} \Delta \boldsymbol{\theta}_{m}^{T} \boldsymbol{\Gamma}_{\theta m}^{-1} \Delta \boldsymbol{\theta}_{m} \\
& +\frac{1}{2} \mathbf{C}_{2} \mathbf{C}_{3}^{-1} \Delta \boldsymbol{\alpha}_{s}^{T} \boldsymbol{\Gamma}_{\alpha s}^{-1} \Delta \boldsymbol{\alpha}_{s}+\frac{1}{2} \mathbf{C}_{2} \mathbf{C}_{3}^{-1} \Delta \boldsymbol{\theta}_{s}^{T} \boldsymbol{\Gamma}_{\theta s}^{-1} \Delta \boldsymbol{\theta}_{s}
\end{aligned}
$$

where $\Delta \boldsymbol{\theta}_{m}=\hat{\boldsymbol{\theta}}_{m}-\boldsymbol{\theta}_{m}$ and $\Delta \boldsymbol{\theta}_{s}=\hat{\boldsymbol{\theta}}_{s}-\boldsymbol{\theta}_{s}$. Differentiating $V$ along the trajectory of the unified closed-loop system (25) and using Property 2 gives

$$
\begin{aligned}
\dot{V}= & -\mathbf{s}_{m}^{T}\left(\mathbf{K}_{m}+\mathbf{C}_{2} C_{3}^{-1} \mathbf{K}_{s}\right) \mathbf{s}_{m}+\mathbf{s}_{m}^{T} \mathbf{Y}_{m r} \Delta \boldsymbol{\alpha}_{m}+\mathbf{s}_{m}^{T}\left(\mathbf{N}_{x m}\left(\mathbf{q}_{m}, \dot{\mathbf{q}}_{m}, \boldsymbol{\eta}_{m}\right)-\mathbf{N}_{x m}\left(\mathbf{q}_{m}, \dot{\mathbf{q}}_{m}, \mathbf{0}\right)-\mathbf{Q}_{m} \hat{\boldsymbol{\theta}}_{m}\right) \\
& -\mathbf{s}_{m}^{T} \mathbf{C}_{2} \mathbf{C}_{3}^{-1} \mathbf{Y}_{s r} \Delta \boldsymbol{\alpha}_{s}-\mathbf{s}_{m}^{T} \mathbf{C}_{2} \mathbf{C}_{3}^{-1}\left(\mathbf{N}_{x s}\left(\mathbf{q}_{s}, \dot{\mathbf{q}}_{s}, \boldsymbol{\eta}_{s}\right)-\mathbf{N}_{x s}\left(\mathbf{q}_{s}, \dot{\mathbf{q}}_{s}, \mathbf{0}\right)-\mathbf{Q}_{s} \hat{\boldsymbol{\theta}}_{s}\right) \\
& +\Delta \boldsymbol{\alpha}_{m}^{T} \boldsymbol{\Gamma}_{\alpha m}^{-1} \dot{\hat{\boldsymbol{\alpha}}}_{m}+\Delta \boldsymbol{\theta}_{m}^{T} \boldsymbol{\Gamma}_{\theta m}^{-1} \dot{\hat{\boldsymbol{\theta}}}_{m}+\mathbf{C}_{2} \mathbf{C}_{3}^{-1} \Delta \boldsymbol{\alpha}_{s}^{T} \boldsymbol{\Gamma}_{\alpha s}^{-1} \dot{\hat{\boldsymbol{\alpha}}}_{s}+\mathbf{C}_{2} \mathbf{C}_{3}^{-1} \Delta \boldsymbol{\theta}_{s}^{T} \boldsymbol{\Gamma}_{\theta s}^{-1} \dot{\hat{\boldsymbol{\theta}}}_{s}
\end{aligned}
$$

Since $\mathbf{s}_{s}=-\mathbf{s}_{m}$, (31) can be written as

$$
\begin{aligned}
\dot{V}= & -\mathbf{s}_{m}^{T}\left(\mathbf{K}_{m}+\mathbf{C}_{2} \mathbf{C}_{3}^{-1} \mathbf{K}_{s}\right) \mathbf{s}_{m}+\mathbf{s}_{m}^{T} \mathbf{Y}_{m r} \Delta \boldsymbol{\alpha}_{m}+\mathbf{s}_{m}^{T}\left(\mathbf{N}_{x m}\left(\mathbf{q}_{m}, \dot{\mathbf{q}}_{m}, \boldsymbol{\eta}_{m}\right)-\mathbf{N}_{x m}\left(\mathbf{q}_{m}, \dot{\mathbf{q}}_{m}, \mathbf{0}\right)-\mathbf{Q}_{m} \hat{\boldsymbol{\theta}}_{m}\right) \\
& +\mathbf{s}_{s}^{T} \mathbf{C}_{2} \mathbf{C}_{3}^{-1} \mathbf{Y}_{s r} \Delta \boldsymbol{\alpha}_{s}+\mathbf{s}_{s}^{T} \mathbf{C}_{2} \mathbf{C}_{3}^{-1}\left(\mathbf{N}_{x s}\left(\mathbf{q}_{s}, \dot{\mathbf{q}}_{s}, \boldsymbol{\eta}_{s}\right)-\mathbf{N}_{x s}\left(\mathbf{q}_{s}, \dot{\mathbf{q}}_{s}, \mathbf{0}\right)-\mathbf{Q}_{s} \hat{\boldsymbol{\theta}}_{s}\right) \\
& +\Delta \boldsymbol{\alpha}_{m}^{T} \boldsymbol{\Gamma}_{\alpha m}^{-1} \dot{\hat{\boldsymbol{\alpha}}}_{m}+\Delta \boldsymbol{\theta}_{m}^{T} \boldsymbol{\Gamma}_{\theta m}^{-1} \dot{\hat{\boldsymbol{\theta}}}_{m}+\mathbf{C}_{2} \mathbf{C}_{3}^{-1} \Delta \boldsymbol{\alpha}_{s}^{T} \boldsymbol{\Gamma}_{\alpha s}^{-1} \dot{\boldsymbol{\alpha}}_{s}+\mathbf{C}_{2} \mathbf{C}_{3}^{-1} \Delta \boldsymbol{\theta}_{s}^{T} \boldsymbol{\Gamma}_{\theta s}^{-1} \dot{\hat{\boldsymbol{\theta}}}_{s}
\end{aligned}
$$

Using Lemma 1, (32) becomes

$$
\begin{aligned}
\dot{V} \leq & -\mathbf{s}_{m}^{T}\left(\mathbf{K}_{m}+\mathbf{C}_{2} \mathbf{C}_{3}{ }^{-1} \mathbf{K}_{s}\right) \mathbf{s}_{m}+\mathbf{s}_{m}^{T} \mathbf{Y}_{m r} \Delta \boldsymbol{\alpha}_{m}-\mathbf{s}_{m}^{T} \mathbf{Q}_{m} \Delta \boldsymbol{\theta}_{m} \\
& +\mathbf{s}_{s}^{T} \mathbf{C}_{2} \mathbf{C}_{3}^{-1} \mathbf{Y}_{s r} \Delta \boldsymbol{\alpha}_{s}-\mathbf{s}_{s}^{T} \mathbf{C}_{2} \mathbf{C}_{3}^{-1} \mathbf{Q}_{s} \Delta \boldsymbol{\theta}_{s} \\
& +\Delta \boldsymbol{\alpha}_{m}^{T} \boldsymbol{\Gamma}_{\alpha m}^{-1} \dot{\hat{\boldsymbol{\alpha}}}_{m}+\Delta \boldsymbol{\theta}_{m}^{T} \boldsymbol{\Gamma}_{\theta m}^{-1} \dot{\hat{\boldsymbol{\theta}}}_{m}+\mathbf{C}_{2} \mathbf{C}_{3}^{-1} \Delta \boldsymbol{\alpha}_{s}^{T} \boldsymbol{\Gamma}_{\alpha s}^{-1} \dot{\hat{\boldsymbol{\alpha}}}_{s}+\mathbf{C}_{2} \mathbf{C}_{3}^{-1} \Delta \boldsymbol{\theta}_{s}^{T} \boldsymbol{\Gamma}_{\theta s}^{-1} \dot{\hat{\theta}}_{s}
\end{aligned}
$$

Substituting the update laws (21)-(22) into (33), we obtain

$$
\dot{V} \leq-\mathbf{s}_{m}^{T}\left(\mathbf{K}_{m}+\mathbf{C}_{2} \mathbf{C}_{3}^{-1} \mathbf{K}_{s}\right) \mathbf{s}_{m}
$$

From (30) and (34), we know that $V$ is positive-definite and $\dot{V}$ is negative semi-definite. Therefore, $V$ is decreasing and bounded. Hence, the signals $\mathbf{s}_{m}, \Delta \boldsymbol{\alpha}_{m}, \Delta \boldsymbol{\theta}_{m}, \Delta \boldsymbol{\alpha}_{s}$ and $\Delta \boldsymbol{\theta}_{s}$ are bounded. 
In terms of position tracking, the definition (11) of $\mathbf{s}_{m}=\Delta \dot{\mathbf{x}}_{m}+\lambda \Delta \mathbf{x}_{m}$ represents a stable first-order system in $\Delta \mathbf{x}_{m}$ with $\mathbf{s}_{m}$ as an input, which has a pole at $-\lambda$ in the left-half of the complex plane as $\lambda$ is positive definite. Thus, the boundedness of $\mathbf{s}_{m}$ amounts to the boundedness of $\Delta \dot{\mathbf{x}}_{m}$ and $\Delta \mathbf{x}_{m}$. Moreover, from (34), we have

$$
\begin{aligned}
& e i g_{\min }\left(\mathbf{K}_{m}\right) \mathbf{s}_{m}^{T} \mathbf{s}_{m}+e i g_{\min }\left(\mathbf{C}_{2} \mathbf{C}_{3} \mathbf{K}_{s}\right) \mathbf{s}_{m}^{T} \mathbf{s}_{m} \\
\leq & \mathbf{s}_{m}^{T}\left(\mathbf{K}_{m}+\mathbf{C}_{2} \mathbf{C}_{3}^{-1} \mathbf{K}_{s}\right) \mathbf{s}_{m} \leq-\dot{V}
\end{aligned}
$$

Integrating both sides of (35), we get

$$
\begin{aligned}
& e i g_{\min }\left(\mathbf{K}_{m}\right) \int_{0}^{t}\left\|\mathbf{s}_{m}\right\|^{2} d t+e i g_{\min }\left(\mathbf{C}_{2} \mathbf{C}_{3} \mathbf{K}_{s}\right) \int_{0}^{t}\left\|\mathbf{s}_{s}\right\|^{2} d t \\
\leq & -\int_{0}^{t} \dot{V} d t=V(0)-V(t) \leq V(0)<\infty
\end{aligned}
$$

where $e i g_{\min }\left(\mathbf{K}_{m}\right)$ and $e i g_{\min }\left(\mathbf{C}_{2} \mathbf{C}_{3} \mathbf{K}_{s}\right)$ denote the minimum eigenvalues of matrices $\mathbf{K}_{m}$ and $\mathbf{C}_{2} \mathbf{C}_{3} \mathbf{K}_{s}$, respectively. Hence, $\mathbf{s}_{m}=\Delta \dot{\mathbf{x}}_{m}+\lambda \Delta \mathbf{x}_{m} \in L_{2}$, which is followed by $\Delta \dot{\mathbf{x}}_{m}, \Delta \mathbf{x}_{m} \in L_{2}$. Thus, according to Lemma 2, we have $\Delta \mathbf{x}_{m} \rightarrow 0$ as $t \rightarrow \infty$.

In terms of force tracking, we already have that $\mathbf{s}_{m}, \Delta \boldsymbol{\alpha}_{m}, \Delta \boldsymbol{\theta}_{m}, \Delta \boldsymbol{\alpha}_{s}, \Delta \boldsymbol{\theta}_{s}$ are bounded and, according to the unified closed loop (25) and Lemma 1, we get

$$
\begin{aligned}
& \left(\mathbf{M}_{x m}\left(\mathbf{q}_{m}\right)+\mathbf{C}_{2} \mathbf{C}_{3}^{-1} \mathbf{M}_{x s}\left(\mathbf{q}_{s}\right)\right) \dot{\mathbf{s}}_{m} \\
\leq & -\left(\mathbf{C}_{x m}\left(\mathbf{q}_{m}, \dot{\mathbf{q}}_{m}\right)+\mathbf{C}_{2} \mathbf{C}_{3}^{-1} \mathbf{C}_{x s}\left(\mathbf{q}_{s}\right)\right) \mathbf{s}_{m}-\left(\mathbf{K}_{m}+\mathbf{C}_{2} \mathbf{C}_{3}{ }^{-1} \mathbf{K}_{s}\right) \mathbf{s}_{m} \\
& +\mathbf{Y}_{m r} \Delta \boldsymbol{\alpha}_{m}-\mathbf{Q}_{m} \Delta \boldsymbol{\theta}_{m}-\mathbf{C}_{2} \mathbf{C}_{3}{ }^{-1} \mathbf{Y}_{s r} \Delta \boldsymbol{\alpha}_{s}+\mathbf{C}_{2} \mathbf{C}_{3}{ }^{-1} \mathbf{Q}_{s} \Delta \boldsymbol{\theta}_{s}
\end{aligned}
$$

Thus, $\dot{\mathbf{s}}_{m}$ is bounded. Furthermore, according to (23) and Lemma 1, we have

$$
\mathbf{C}_{2}\left(\mathbf{f}_{h}-\mathbf{f}_{e}\right) \leq \mathbf{M}_{x m}\left(\mathbf{q}_{m}\right) \dot{\mathbf{s}}_{m}+\mathbf{C}_{x m}\left(\mathbf{q}_{m}, \dot{\mathbf{q}}_{m}\right) \mathbf{s}_{m}+\mathbf{K}_{m} \mathbf{s}_{m}-\mathbf{Y}_{m r} \Delta \boldsymbol{\alpha}_{m}+\mathbf{Q}_{m} \Delta \boldsymbol{\theta}_{m}
$$

Therefore, $\Delta \mathbf{f}=\mathbf{f}_{h}-\mathbf{f}_{e}$ is bounded as $t \rightarrow \infty$. This concludes the proof.

Remark 4: It is worth noting that in adaptive control, the tracking error should converge regardless of whether the input is persistently exciting or not, i.e., model parameter convergence should not be a prerequisite for tracking error convergence. As can be seen from the proof of Theorem 1, position tracking error converges to zero as $t \rightarrow \infty$, and the force tracking error can remain bounded regardless of the convergence of model parameters $\boldsymbol{\alpha}_{m}, \boldsymbol{\alpha}_{s}, \boldsymbol{\theta}_{m}$, and $\boldsymbol{\theta}_{s}$. 


\section{Examples of NLP Terms in Robot Dynamics}

In this section, we consider the two-degree-of-freedom revolute-prismatic (RP) robot shown in Fig. 3 to illustrate two typical examples that involve NLP dynamic terms. The joint-space dynamics of the RP robot is expressed as [25]

$$
\mathbf{M}(\mathbf{q}) \ddot{\mathbf{q}}+\mathbf{C}(\mathbf{q}, \dot{\mathbf{q}}) \dot{\mathbf{q}}+\mathbf{G}(\mathbf{q})=\boldsymbol{\tau}
$$

where

$$
\begin{aligned}
& \mathbf{M}(\mathbf{q})=\left[\begin{array}{cc}
m_{1} l_{1}^{2}+m_{2} d_{2}^{2} & 0 \\
0 & m_{2}
\end{array}\right], \quad \mathbf{C}(\mathbf{q}, \dot{\mathbf{q}})=\left[\begin{array}{cc}
0 & 2 m_{2} d_{2} \dot{q}_{1} \\
-m_{2} d_{2} \dot{q}_{1} & 0
\end{array}\right], \\
& \mathbf{G}(\mathbf{q})=\left[\begin{array}{c}
\left(m_{1} l_{1}+m_{2} d_{2}\right) g \cos \left(q_{1}\right) \\
m_{2} g \sin \left(q_{1}\right)
\end{array}\right], \\
& \mathbf{J}=\left[\begin{array}{cc}
-d_{2} \sin q_{1} & \cos q_{1} \\
d_{2} \cos q_{1} & \sin q_{1}
\end{array}\right]=\left[\begin{array}{cc}
-\left(l_{1}+d_{1}\right) \sin q_{1} & \cos q_{1} \\
\left(l_{1}+d_{1}\right) \cos q_{1} & \sin q_{1}
\end{array}\right],
\end{aligned}
$$

where $l_{1}$ is the length of the first link, $m_{1}$ and $m_{2}$ are the point masses of the two links, $g$ is the gravity coefficient, $q_{1}$ is a variable angle corresponding to the position of the rotary joint, $d_{1}$ is a variable distance corresponding to the position of the prismatic joint, $d_{2}$ is the total length of the robot, and $\mathbf{q}=\left[q_{1}, d_{2}\right]^{T}$.

\section{Example 1. Stribeck effect of friction}

A typical NLP structure is the Stribeck effect of friction torques at robot joints $q_{1}$ and $d_{2}$, where the NLP part in the joint space is modeled as [28]

$$
N_{i}=\left(\mu_{s i}-\mu_{c i}\right) \operatorname{sgn}\left(\dot{q}_{i}\right) \exp \left(-\frac{\dot{q}_{i}^{2}}{v_{s i}^{2}}\right)
$$

Here, $i$ denotes the $i^{\text {th }}$ joint, $\mu_{s i}$ is the static friction coefficient, $\mu_{c i}$ is the Coulomb friction coefficient, and $v_{s i}$ is the Stribeck velocity parameter.

For the NLP joint-level friction torque term in (41), according to (6), we get that the friction force of the entire robot in Cartesian space as 


$$
\begin{aligned}
\mathbf{N}_{x} & =\mathbf{J}^{-T} \mathbf{N}=\mathbf{J}^{-T}\left[\begin{array}{l}
N_{1} \\
N_{2}
\end{array}\right] \\
& =\left[\begin{array}{l}
\frac{\sin q_{1}}{d_{2}}\left(\mu_{s 1}-\mu_{c 1}\right) \operatorname{sign}\left(\dot{q}_{1}\right) \exp \left(-\frac{\dot{q}_{1}^{2}}{v_{s 1}^{2}}\right)+\cos \left(q_{1}\right)\left(F_{s 2}-F_{c 2}\right) \operatorname{sign}\left(\dot{d}_{2}\right) \exp \left(-\frac{\dot{d}_{2}^{2}}{v_{s 2}^{2}}\right) \\
\frac{\cos q_{1}}{d_{2}}\left(\mu_{s 1}-\mu_{c 1}\right) \operatorname{sign}\left(\dot{q}_{1}\right) \exp \left(-\frac{\dot{q}_{1}^{2}}{v_{s 1}^{2}}\right)+\sin \left(q_{1}\right)\left(F_{s 2}-F_{c 2}\right) \operatorname{sign}\left(\dot{d}_{2}\right) \exp \left(-\frac{\dot{d}_{2}^{2}}{v_{s 2}^{2}}\right)
\end{array}\right]
\end{aligned}
$$

As can be seen, the Stribeck velocity parameter $v_{s 1}$ and $v_{s 2}$ appear nonlinearly in (42), and $\mathbf{N}_{x}$ cannot be linearized as in Property 1, i.e., $\mathbf{N}_{x}$ cannot be written as the product $\mathbf{Y} \boldsymbol{\alpha}$ where $q_{1}$ and $d_{2}$ are included in the regressor matrix $\mathbf{Y}$, and $v_{s 1}$ and $v_{s 2}$ are included in the unknown parameter vector $\boldsymbol{\alpha}$.Thus, the Stribeck effect of friction is a NLP term.

\section{Example 2. Effect of kinematic terms in Cartesian-space dynamics}

Another typical example on NLP dynamic terms is how kinematic parameters enter the robot dynamics in Cartesian space. It is well known that robot kinematic parameters are not linearly parameterized in Cartesian space models. That is, Property 1 is only valid for dynamic uncertainties in general. This is due to the presence of inverse Jacobian in the Cartesian space model. For most robots, it is not possible to extract out the uncertain kinematic parameters in the linear fashion of Property 1 . Therefore, kinematic uncertainties involve NLP structures in Cartesian space.

According to the joint-space dynamics of the RP robot (39)-(40) and the space transformation (6), we can get the dynamics of the RP in Cartesian space as

$$
\mathbf{M}_{x}=\left[\begin{array}{cc}
-\frac{-m_{1} l_{1}^{2}+\cos \left(q_{1}^{2}\right) m_{1} l_{1}^{2}}{\left(l_{1}+d_{1}\right)^{2}}+m_{2} & -\frac{\sin \left(q_{1}\right) \cos \left(q_{1}\right) m_{1} l_{1}^{2}}{\left(l_{1}+d_{1}\right)^{2}} \\
-\frac{\sin \left(q_{1}\right) \cos \left(q_{1}\right) m_{1} l_{1}^{2}}{\left(l_{1}+d_{1}\right)^{2}} & \frac{\cos \left(q_{1}^{2}\right) m_{1} l_{1}^{2}}{\left(l_{1}+d_{1}\right)^{2}}+m_{2}
\end{array}\right],
$$




$$
\begin{gathered}
\mathbf{C}_{x}=\left[\begin{array}{cc}
\frac{\cos \left(q_{1}\right) \sin \left(q_{1}\right) \dot{q}_{1} m_{1} l_{1}^{2}}{\left(l_{1}+d_{1}\right)^{2}}-\cos \left(q_{1}\right) \sin \left(q_{1}\right) \dot{q}_{1} m_{2} & -\frac{\left(-1+\cos \left(q_{1}^{2}\right)\right) \dot{q}_{1} m_{1} l_{1}^{2}}{\left(l_{1}+d_{1}\right)^{2}}+\left(-1+\cos \left(q_{1}^{2}\right)\right) \dot{q}_{1} m_{2} \\
-\frac{\cos \left(q_{1}^{2}\right) \dot{q}_{1} m_{1} l_{1}^{2}}{\left(l_{1}+d_{1}\right)^{2}}+\cos \left(q_{1}^{2}\right) \dot{q}_{1} m_{2} & -\frac{\cos \left(q_{1}\right) \sin \left(q_{1}\right) \dot{q}_{1} m_{1} l_{1}^{2}}{\left(l_{1}+d_{1}\right)^{2}}+\cos \left(q_{1}\right) \sin \left(q_{1}\right) m_{2} \dot{q}_{1}
\end{array}\right], \\
\mathbf{G}_{x}=\left[\begin{array}{l}
-\frac{\sin \left(q_{1}\right) \cos \left(q_{1}\right) m_{1} l_{1} g}{l_{1}+d_{1}} \\
\frac{\left(\cos \left(q_{1}\right)^{2} m_{1} l_{1}\right.}{l_{1}+d_{1}}+m_{2} g
\end{array}\right] .
\end{gathered}
$$

It can be seen that the kinematic parameter $l_{1}$ appears nonlinearly in $\mathbf{M}_{x}, \mathbf{C}_{x}$ and $\mathbf{G}_{x}$. Also, unlike Property

1, (43) cannot be written as the product $\mathbf{Y} \boldsymbol{\alpha}$ such that the joint positions $q_{1}$ and $d_{1}$ are included in the regressor matrix $\mathbf{Y}$ and the kinematic parameter $l_{1}$ is included in the parameter vector $\boldsymbol{\alpha}$. In other words, the terms involving kinematic parameters in Cartesian space dynamics are NLP terms for the RP robot.

As coping with kinematic uncertainties is out of the scope of this paper, we focus on the dynamic uncertainties and further pursue the example of NLP terms originating from Stribeck friction in the simulation studies in the next section.

\section{Simulation Studies}

In this section, simulations are done to demonstrate the validity of the proposed adaptive control scheme.

We take two identical RP manipulators similar to the one shown in Fig. 3 as the master and the slave robots.

The LP dynamic parameter vectors $\boldsymbol{\alpha}_{m}, \boldsymbol{\alpha}_{s}$ can be found as $\boldsymbol{\alpha}_{m}=\boldsymbol{\alpha}_{s}=\left[m_{1} l_{1}^{2}, m_{2}, m_{1} l_{1} g, m_{2} g\right]^{T}$. Then, according to Property 1, the regressor matrices $\mathbf{Y}_{m r}, \mathbf{Y}_{s r}$ can be found. The calculation of $\boldsymbol{\alpha}_{m}, \boldsymbol{\alpha}_{s}$ and $\mathbf{Y}_{m r}, \mathbf{Y}_{s r}$ can be found in Appendix A.

For the NLP term, we consider the Stribeck friction at the robot joints [28]-[29]. For simplicity and without loss of generality, we ignore the LP part in the friction model and focus on the NLP part shown in (41) in Example 1. The calculation of the parameter vectors $\boldsymbol{\theta}_{m}, \boldsymbol{\theta}_{s}$, and the related matrices $\mathbf{Q}_{m}, \mathbf{Q}_{s}$ can be found in Appendix B. In the simulations, the exogenous force from the human operator is chosen as $\mathbf{f}_{h}^{*}=[\sin t, 0]^{T}$ and 
the selected robot parameters, friction parameters (adopted from [23]), and controller parameters are shown in Table 1.

According to Table 1, the actual values of the LP uncertain vectors are obtained as $\boldsymbol{\alpha}_{m}=\boldsymbol{\alpha}_{s}=[1.15,2.3,22.54,22.54]^{T}$, and the actual values of the NLP uncertain vectors are $\boldsymbol{\theta}_{m}=\boldsymbol{\theta}_{s}=[3845.2,62,3845.2,62]^{T}$. In the simulations, the robots initial positions were set as $\mathbf{x}_{m}(0)=\mathbf{x}_{s}(0)=[0.4,0.6928]^{T}$, and the initial estimates of the uncertain parameter vectors were randomly chosen, i.e., some initial estimates were lower than the actual values and some initial estimates were higher than the actual values. Indeed, the initial estimates of $\boldsymbol{\alpha}_{m}$ and $\boldsymbol{\alpha}_{s}$ were $\hat{\boldsymbol{\alpha}}_{m}(0)=\hat{\boldsymbol{\alpha}}_{s}(0)=[0.95,2.7,19,25]^{T}$, which correspond to -17.39 to $+17.39 \%$ deviation from the nominal values, and the initial estimates of $\boldsymbol{\theta}_{m}$ and $\boldsymbol{\theta}_{s}$ were taken as $\hat{\boldsymbol{\theta}}_{m}(0)=\hat{\boldsymbol{\theta}}_{s}(0)=[4000,60,3600,70]^{T}$, which correspond to $-6.38 \%$ to $+12.9 \%$ deviation from the nominal values.

When the system simultaneously has LP dynamic uncertainties (i.e., uncertain $\boldsymbol{\alpha}_{m}$ and $\boldsymbol{\alpha}_{s}$ ) and NLP dynamic uncertainties (i.e., uncertain $\boldsymbol{\theta}_{m}$ and $\boldsymbol{\theta}_{s}$ ), the proposed adaptive control scheme is compared with a conventional adaptive control scheme [16], which can only deal with LP dynamic uncertainties, given as

$$
\begin{aligned}
& \mathbf{f}_{m}=-\mathbf{K}_{m} \mathbf{s}_{m}+\mathbf{Y}_{m r} \hat{\boldsymbol{\alpha}}_{m}+\mathbf{C}_{2}\left(\mathbf{f}_{h}-\mathbf{f}_{e}\right)-\mathbf{f}_{h} \\
& \mathbf{f}_{s}=-\mathbf{K}_{s} \mathbf{s}_{s}+\mathbf{Y}_{s r} \hat{\boldsymbol{\alpha}}_{s}+\mathbf{C}_{3}\left(\mathbf{f}_{h}-\mathbf{f}_{e}\right)+\mathbf{f}_{e} \\
& \dot{\hat{\boldsymbol{\alpha}}}_{m}=-\boldsymbol{\Gamma}_{a m} \mathbf{Y}_{m r}^{T} \mathbf{s}_{m}, \dot{\hat{\boldsymbol{\alpha}}}_{s}=-\boldsymbol{\Gamma}_{a s} \mathbf{Y}_{s r}^{T} \mathbf{s}_{s}
\end{aligned}
$$

and with the fixed 4-channel control scheme, which is not meant to deal with any uncertainties, given as

$$
\begin{aligned}
& \mathbf{f}_{m}=-\mathbf{K}_{m} \mathbf{s}_{m}+\mathbf{C}_{2}\left(\mathbf{f}_{h}-\mathbf{f}_{e}\right)-\mathbf{f}_{h} \\
& \mathbf{f}_{s}=-\mathbf{K}_{s} \mathbf{s}_{s}+\mathbf{C}_{3}\left(\mathbf{f}_{h}-\mathbf{f}_{e}\right)+\mathbf{f}_{e}
\end{aligned}
$$

The simulation results in x-direction are shown in Fig. 4 to Fig. 6. From Fig. 4 we can see that, for the fixed control scheme, the position tracking and the force tracking errors are the biggest among these three control schemes as the control method can compensate neither for LP-type uncertainties in $\boldsymbol{\alpha}_{m}, \boldsymbol{\alpha}_{s}$ nor for NLP-type uncertainties in $\boldsymbol{\theta}_{m}$ and $\boldsymbol{\theta}_{s}$. Comparatively, from Fig. 5 we can see that the performance of the conventional 
adaptive control is better than that of the fixed control scheme because it can compensate for the LP uncertainties in $\boldsymbol{\alpha}_{m}$ and $\boldsymbol{\alpha}_{s}$. However, the position tracking of the conventional adaptive control is not perfect yet and the force tracking error is still big as the method is not meant to compensate for the NLP uncertainties in $\boldsymbol{\theta}_{m}$ and $\boldsymbol{\theta}_{s}$. However, from Fig. 6 we can see that for the proposed adaptive control, the position trajectories of the master and the slave are very close to one another, and the force tracking error is bounded and quite small, as the method is able to compensate for LP uncertainties in $\boldsymbol{\alpha}_{m}, \boldsymbol{\alpha}_{s}$, and NLP uncertainties in $\boldsymbol{\theta}_{m}$ and $\boldsymbol{\theta}_{s}$. Since the exogenous force $\mathbf{f}_{h}^{*}$, which acts as the excitation in the simulations, is zero in the y-direction, the position and force profiles present good matches and are not shown in that direction.

\section{Conclusions}

In this paper, for a bilateral teleoperation system, adaptive controllers are designed for the master and slave robots suffering from both linearly parameterized (LP) and nonlinearly parameterized (NLP) dynamic uncertainties. The controllers are incorporated into the 4-channel bilateral teleoperation control framework. Transparency of the overall teleoperation is studied via a Lyapunov function analysis and demonstrated by simulation studies. Compared with a conventional adaptive control scheme, which is not able to deal with NLP dynamic uncertainties, the proposed control scheme demonstrates better position and force tracking performance despite the deviations in the LP and NLP terms in dynamics.

With respect to position tracking, we have ensured that the master-slave position tracking error converges to zero as $t \rightarrow \infty$, while we have shown the force tracking error is bounded. Improving the present control algorithm to ensure that the force tracking error converges to zero remains for future. In addition, kinematic uncertainties and communication time delay are not dealt with in the scope of this paper, and will be addressed in our future work.

\section{Acknowledgements}


This research was supported by the Natural Sciences and Engineering Research Council (NSERC) of Canada under grants RGPIN-372042 and EQPEQ-375712, and by the China Scholarship Council (CSC) under grant [2009]3012.

\section{Appendix A}

Calculations involving LP terms in the RP robot Cartesian-space dynamics

According to the joint-space dynamics of the RP robot (40), the transformation of the dynamics from joint space to Cartesian space (6), and the definitions of $\mathbf{Y}_{r}$ (17)-(18), we can get

$\mathbf{Y}_{r}\left(q, \dot{\mathbf{q}}, \dot{\mathbf{x}}_{r}, \ddot{\mathbf{x}}_{r}\right) \boldsymbol{\alpha}=\mathbf{M}_{x}(\mathbf{q}) \ddot{\mathbf{x}}_{r}+\mathbf{C}_{x}(\mathbf{q}, \dot{\mathbf{q}}) \dot{\mathbf{x}}_{r}+\mathbf{G}_{x}(\mathbf{q})$

$$
\begin{aligned}
& =\left[\begin{array}{cc}
-\frac{-m_{1} l_{1}^{2}+\cos \left(q_{1}^{2}\right) m_{1} l_{1}^{2}}{d_{2}^{2}}+m_{2} & -\frac{\sin \left(q_{1}\right) \cos \left(q_{1}\right) m_{1} l_{1}^{2}}{d_{2}^{2}} \\
-\frac{\sin \left(q_{1}\right) \cos \left(q_{1}\right) m_{1} l_{1}^{2}}{d_{2}^{2}} & \frac{\cos \left(q_{1}^{2}\right) m_{1} l_{1}^{2}}{d_{2}^{2}}+m_{2}
\end{array}\right] \ddot{\mathbf{x}}_{r}+ \\
& {\left[\begin{array}{cc}
\frac{\cos \left(q_{1}\right) \sin \left(q_{1}\right) \dot{q}_{1} m_{1} l_{1}^{2}}{d_{2}^{2}}-\cos \left(q_{1}\right) \sin \left(q_{1}\right) \dot{q}_{1} m_{2} & -\frac{\left(-1+\cos \left(q_{1}^{2}\right)\right) \dot{q}_{1} m_{1} l_{1}^{2}}{d_{2}^{2}}+\left(-1+\cos \left(q_{1}^{2}\right)\right) \dot{q}_{1} m_{2} \\
-\frac{\cos \left(q_{1}^{2}\right) \dot{q}_{1} m_{1} l_{1}^{2}}{d_{2}{ }^{2}}+\cos \left(q_{1}^{2}\right) \dot{q}_{1} m_{2} & -\frac{\cos \left(q_{1}\right) \sin \left(q_{1}\right) \dot{q}_{1} m_{1} l_{1}^{2}}{d_{2}^{2}}+\cos \left(q_{1}\right) \sin \left(q_{1}\right) m_{2} \dot{q}_{1}
\end{array}\right] \dot{\mathbf{x}}_{r}+} \\
& {\left[\begin{array}{c}
-\frac{\sin \left(q_{1}\right) \cos \left(q_{1}\right) m_{1} l_{1} g}{d_{2}} \\
\frac{\left(\cos \left(q_{1}\right)^{2} m_{1} l_{1}\right.}{d_{2}}+m_{2} g
\end{array}\right] .}
\end{aligned}
$$

Simplify the above, and we can obtain $\boldsymbol{\alpha}$ and $\mathbf{Y}_{r}$ as

$$
\boldsymbol{\alpha}=\left[m_{1} l_{1}^{2}, m_{2}, m_{1} l_{1} g, m_{2} g\right]^{T},
$$




$$
\begin{aligned}
& \mathbf{Y}_{r}=\left[\begin{array}{llll}
\frac{\ddot{x}_{r 1}-\ddot{x}_{r 1} \cos \left(2 q_{1}\right)-\ddot{x}_{r 2} \sin \left(2 q_{1}\right)}{2 d_{2}^{2}} & \ddot{x}_{r 1} & 0 & 0 \\
\frac{\ddot{x}_{r 2}-\ddot{x}_{r 1} \sin \left(2 q_{1}\right)+\ddot{x}_{r 2} \cos \left(2 q_{1}\right)}{2 d_{2}^{2}} & \ddot{x}_{r 2} & 0 & 0
\end{array}\right]+ \\
& {\left[\begin{array}{llll}
\frac{\dot{q}_{1} \dot{x}_{r 1} \sin \left(2 q_{1}\right)+\dot{q}_{1} \dot{x}_{r 2}-\dot{q}_{1} \dot{x}_{r 2} \cos \left(2 q_{1}\right)}{2 d_{2}{ }^{2}} & \frac{-\dot{q}_{1} d_{2}{ }^{2} \dot{x}_{r 1} \sin \left(2 q_{1}\right)-\dot{q}_{1} d_{2}{ }^{2} \dot{x}_{r 2}+\dot{q}_{1} d_{2}{ }^{2} \dot{x}_{r 2} \cos \left(2 q_{1}\right)}{2 d_{2}{ }^{2}} & 0 & 0 \\
\frac{-\dot{q}_{1} \dot{x}_{r 1} \cos \left(2 q_{1}\right)-\dot{q}_{1} \dot{x}_{r 1}-\dot{q}_{1} \dot{x}_{r 2} \sin \left(2 q_{1}\right)}{2 d_{2}{ }^{2}} & \frac{\dot{q}_{1} d_{2}{ }^{2} \dot{x}_{r 1} \cos \left(2 q_{1}\right)+\dot{q}_{1} d_{2}{ }^{2} \dot{x}_{r 1}+\dot{q}_{1} d_{2}{ }^{2} \dot{x}_{r 2} \sin \left(2 q_{1}\right)}{2 d_{2}{ }^{2}} & 0 & 0
\end{array}\right]+} \\
& {\left[\begin{array}{cccc}
0 & 0 & \frac{-\sin \left(q_{1}\right) \cos \left(q_{1}\right)}{d_{2}} & 0 \\
0 & 0 & \frac{\cos \left(q_{1}^{2}\right)}{d_{2}} & 1
\end{array}\right],}
\end{aligned}
$$

where $x_{r 1}$ and $x_{r 2}$ are the two elements of $\mathbf{x}_{r}$, i.e., $\mathbf{x}_{r}=\left[x_{r 1}, x_{r 2}\right]^{T}$. Hereafter, we omit for simplicity the subscripts $m$ and $s$ in the variables for the master and for the slave.

\section{Appendix B}

Calculations involving friction-induced NLP terms in the RP robot Cartesian-space dynamics

In Section 4, for the NLP friction torque (41), we get the corresponding force in Cartesian space in (42) as

$$
\mathbf{N}_{x}=\left[\begin{array}{l}
-\frac{\sin \left(q_{1}\right)}{d_{2}}\left(\mu_{s 1}-\mu_{c 1}\right) \operatorname{sign}\left(\dot{q}_{1}\right) \exp \left(-\frac{\dot{q}_{1}^{2}}{v_{s 1}^{2}}\right)+\cos \left(q_{1}\right)\left(F_{s 2}-F_{c 2}\right) \operatorname{sign}\left(\dot{d}_{2}\right) \exp \left(-\frac{\dot{d}_{2}^{2}}{v_{s 2}^{2}}\right) \\
\frac{\cos \left(q_{1}\right)}{d_{2}}\left(\mu_{s 1}-\mu_{c 1}\right) \operatorname{sign}\left(\dot{q}_{1}\right) \exp \left(-\frac{\dot{q}_{1}^{2}}{v_{s 1}^{2}}\right)+\sin \left(q_{1}\right)\left(F_{s 2}-F_{c 2}\right) \operatorname{sign}\left(\dot{d}_{2}\right) \exp \left(-\frac{\dot{d}_{2}^{2}}{v_{s 2}^{2}}\right)
\end{array}\right] .
$$

According to the multiplicative form in (7), we have

$$
\begin{gathered}
\mathbf{R}=\left[\begin{array}{l}
\mathbf{R}_{1} \\
\mathbf{R}_{2}
\end{array}\right]=\left[\begin{array}{cc}
-\frac{\sin \left(q_{1}\right)}{d_{2}}\left(\mu_{s 1}-\mu_{c 1}\right) & \cos \left(q_{1}\right)\left(\mu_{s 2}-\mu_{c 2}\right) \\
\frac{\cos \left(q_{1}\right)}{d_{2}}\left(\mu_{s 1}-\mu_{c 1}\right) & \sin \left(q_{1}\right)\left(\mu_{s 2}-\mu_{c 2}\right)
\end{array}\right], \\
\mathbf{T}=\mathbf{T}_{1}=\mathbf{T}_{2}=\left[\begin{array}{c}
\operatorname{sign}\left(\dot{q}_{1}\right) \exp \left(-\frac{\dot{q}_{1}^{2}}{v_{s 1}^{2}}\right) \\
\operatorname{sign}\left(\dot{d}_{2}\right) \exp \left(-\frac{\dot{d}_{2}^{2}}{v_{s 2}^{2}}\right)
\end{array}\right], \boldsymbol{\eta}=\mathbf{\eta}_{1}=\mathbf{\eta}_{2}=\left[\begin{array}{c}
\mu_{s 2}-\mu_{c 1} \\
\frac{1}{\nu_{s 1}^{2}} \\
\frac{1}{v_{s 2}^{2}}
\end{array}\right] .
\end{gathered}
$$

Xia Liu, DS-10-1274, 20 
Since $\mathbf{R}_{1}, \mathbf{T}_{1}$ are Lipschitz in $\boldsymbol{\eta}_{1}$ and $\mathbf{R}_{2}, \mathbf{T}_{2}$ are Lipschitz in $\boldsymbol{\eta}_{2}$, the related variables in Property 3 and Lemma 1 are obtained as

$$
\begin{gathered}
A_{11}=\left|\frac{\sin \left(q_{1}\right)}{d_{2}}\right|, A_{12}=\left|\cos \left(q_{1}\right)\right|, A_{13}=0, A_{14}=0, \\
A_{21}=\left|\frac{\cos \left(q_{1}\right)}{d_{2}}\right|, A_{22}=\left|\sin \left(q_{1}\right)\right|, A_{23}=0, A_{24}=0, \\
Z_{11}=Z_{12}=Z_{21}=Z_{22}=0, Z_{13}=Z_{23}=\dot{q}_{1}^{2}, Z_{14}=Z_{24}=\dot{d}_{2}^{2} .
\end{gathered}
$$

Then, according to the definitions of $A_{i}$ and $Z_{i}$ in Property 3, we have

$$
\begin{gathered}
A_{1}=\max \left(A_{11}, A_{12}, A_{13}, A_{14}\right)=\max \left(\left|\frac{\sin \left(q_{1}\right)}{d_{2}}\right|, \cos \left(q_{1}\right), 0,0\right), \\
A_{2}=\max \left(A_{21}, A_{22}, A_{23}, A_{24}\right) \max \left(\left|\frac{\cos \left(q_{1}\right)}{d_{2}}\right|, \sin \left(q_{1}\right), 0,0\right), \\
Z_{1}=Z_{2}=\max \left(0,0, \dot{q}_{1}^{2}, \dot{d}_{2}^{2}\right) .
\end{gathered}
$$

Furthermore, according to the definitions of $\boldsymbol{\theta}, \mathbf{w}, \mathbf{Q}$ in Lemma 1, we also have

$$
\begin{aligned}
& \boldsymbol{\theta}_{1}=\boldsymbol{\theta}_{2}=\left[\begin{array}{c}
\left(\mu_{s 1}-\mu_{c 1}+\mu_{s 2}-\mu_{c 2}+\frac{1}{v_{s 1}^{2}}+\frac{1}{v_{s 2}^{2}}\right)^{2} \\
\mu_{s 1}-\mu_{c 1}+\mu_{s 2}-\mu_{c 2}+\frac{1}{v_{s 1}^{2}}+\frac{1}{v_{s 2}^{2}}
\end{array}\right], \\
& \boldsymbol{\theta}=\left[\begin{array}{c}
\left(\mu_{s 1}-\mu_{c 1}+\mu_{s 2}-\mu_{c 2}+\frac{1}{v_{s 1}^{2}}+\frac{1}{v_{s 2}^{2}}\right)^{2} \\
\mu_{s 1}-\mu_{c 1}+\mu_{s 2}-\mu_{c 2}+\frac{1}{v_{s 1}^{2}}+\frac{1}{v_{s 2}^{2}} \\
\left(\mu_{s 1}-\mu_{c 1}+\mu_{s 2}-\mu_{c 2}+\frac{1}{v_{s 1}^{2}}+\frac{1}{v_{s 2}^{2}}\right)^{2} \\
\mu_{s 1}-\mu_{c 1}+\mu_{s 2}-\mu_{c 2}+\frac{1}{v_{s 1}^{2}}+\frac{1}{v_{s 2}^{2}}
\end{array}\right], \\
& \mathbf{w}_{1}=\left[\begin{array}{ll}
A_{1} Z_{1} & \sqrt{2} A_{1}
\end{array}\right], \mathbf{w}_{2}=\left[\begin{array}{ll}
A_{2} Z_{2} & \sqrt{2} A_{2}
\end{array}\right], \\
& \mathbf{Q}=\left[\begin{array}{ccc}
\operatorname{sgn}\left(s_{1}\right) \mathbf{w}_{1} & 0 & 0 \\
0 & 0 & \operatorname{sgn}\left(s_{2}\right) \mathbf{w}_{2}
\end{array}\right]=\left[\begin{array}{cccc}
\mathrm{s} g n\left(s_{1}\right) A_{1} Z_{1} & \sqrt{2} \mathrm{~s} g n\left(s_{1}\right) A_{1} & 0 & 0 \\
0 & 0 & \mathrm{~s} g n\left(s_{2}\right) A_{2} Z_{2} & \sqrt{2} \mathrm{~s} g n\left(s_{2}\right) A_{2}
\end{array}\right] .
\end{aligned}
$$

Xia Liu, DS-10-1274, 21 


\section{References}

[1] Yoon, W. K., Goshozono, T., Kawabe, H., Kinami, M., Tsumaki, Y., Uchiyama, M., Oda, M., and Doi, T., 2004, "Model-based space robot teleoperation of ETS-VII Manipulator,” IEEE Transactions on Robotics and Automation, 20 (3), pp. 602-612.

[2] Hirabayashi, T., Akizono, J., Yamamoto, T., Sakai, H., and Yano, H., 2006, "Teleoperation of construction machines with haptic information for underwater applications," Automation in Construction, 15 (5), pp. 563-570.

[3] Parker, N. R., Salcudean, S.E., and Lawrence, P.D., 1993, "Application of force feedback to heavy duty hydraulic machines", Proceeding of the IEEE International Conference on Robot Automation, Atlanta, GA, USA, pp. 375-381.

[4] Tavakoli, M., Aziminejad, A., Patel, R.V., and Moallem, M., 2006, "Methods and mechanisms for contact feedback in a robot-assisted minimally invasive environment", Surgical Endoscopy and Other Interventional Techniques, 10 (20), pp. 1570-1579.

[5] Wang, W., and Yuan, K., 2004, "Teleoperated manipulator for leak detection of sealed radioactive sources,” IEEE International Conference on Robotics and Automation, New Orleans, LA, pp. 1682-1687.

[6] Melchiorri, C., 2003, "Robotic telemanipulation systems: An overview on control aspects," Proceeding of 7th IFAC Symposium on Robot Control, Wroclaw, Poland, pp. 707-716.

[7] Fite, K. B., Speich, J. E., and Goldfarb, M., 2001, "Transparency and stability robustness in two-channel bilateral telemanipulation,” Journal of Dynamic Systems, Measurement and Control, Transactions of the ASME, 123 (3), pp. 400-407.

[8] Munir, S., and Book, W. J., 2003, "Control techniques and programming issues for time delayed internet based teleoperation,” Journal of Dynamic Systems, Measurement and Control, Transactions of the ASME, 125 (2), pp. 205-214.

[9] Alfi, A., and Farrokhi, M., 2008 "A simple structure for bilateral transparent teleoperation systems with time delay," Journal of Dynamic Systems, Measurement and Control, Transactions of the ASME, 130 (4), pp. 0445021-0445029. 
[10] Polushin, I. G., Liu, P. X., Lung, C.-H., and On, G.D., 2010, "Position-error based schemes for bilateral teleoperation with time delay: theory and experiments," Journal of Dynamic Systems, Measurement and Control, Transactions of the ASME, 132 (3), pp. 1-11.

[11] Hokayem, P., and Spong, M., 2006, "Bilateral teleoperation: An historical survey," Automatica, 42 (12), pp. 2035-2057.

[12] Lawrence, D. A., 1993, "Stability and transparency in bilateral teleoperation," IEEE Transactions on Robotics and Automation, 9 (5), pp. 624-637.

[13] Yokokohji, Y., and Yoshikawa, T., 1994, "Bilateral control of master-slave manipulator for ideal kinesthetic coupling-formulation and experiment," IEEE Transactions on Robotics and Automation, 10 (5), pp. 605-619.

[14] Hyoung-Ki Lee and Chung, M. J., 1998, "Adaptive controller of a master-slave system for transparent teleoperation,” Journal of Robotic systems, 15 (8), pp. 465-475.

[15] Shi, M., Tao, G., and Liu, H., 2002, "Adaptive control of teleoperation systems," Journal of X-Ray Science and Technology, 10 (1-2), pp. 37-57.

[16] Ryu, J. H., and Kwon, D. S., 2001, “A novel adaptive bilateral control scheme using similar closed-loop dynamic characteristics of master/slave manipulators," Journal of Robotic Systems, 18 (9), pp. 533-543.

[17] Hung, N. V. Q., Narikiyo, T., and Tuan, H. D., 2003, "Nonlinear adaptive control of master-slave system in teleoperation," Control Engineering Practice, 11(1), pp. 1-10.

[18] Chopra, N., Spong, M. W., and Lozano, R., 2008, "Synchronization of bilateral teleoperators with time delay,” Automatica, 44 (8), pp. 2142-2148.

[19] Nuño, E., Ortega, R., and Basañez, L., 2010, "An adaptive controller for nonlinear teleoperators," Automatica, 46 (1), pp. 155-159.

[20] Wen-Hong Zhu, and Salcudean, S. E., 2000, "Stability guaranteed teleoperation: An adaptive motion/force control approach," IEEE transactions on automatic control, 45 (11), pp. 1951-1969.

[21] Malysz, P., and Sirouspour, S., 2009, "Nonlinear and filtered force/position mapping in bilateral teleoperation with application to enhanced stiffness discrimination," IEEE Transactions on Robotics, 25 (5), pp. 1134-1149. 
[22] Feemster, M., Vedagarbha, P., Dawson, D. M., and D. Haste, 1999, “Adaptive control techniques for friction compensation," Mechatronics, 9(2), pp.125-145.

[23] Hung, N. V. Q., Tuan, H. D., Narikiyo, T., and Apkarian, P., 2008, “Adaptive control for nonlinearly parameterized uncertainties in robot," IEEE transactions on control systems technology, 16(3), pp.458-468.

[24] Marquez, H. J., 2003, Nonlinear Control Systems, John Wiley \& Sons, Inc., Hoboken, NJ.

[25] Craig, J., 2005, Introduction to Robotics: Mechanics and Control ( ${ }^{\text {rd }}$ Ed.), Pearson Prentice Hall, USA.

[26] Slotine, J. J. E., and Li, W., 1991, Applied Nonlinear Control, Prentice-Hall, Englewood Cliffs, NJ.

[27] Tavakoli, Aziminejad, M., A., Patel, R.V., 2007, and Moallem, M., "High-fidelity bilateral teleoperation systems and the effect of multimodal haptics," IEEE Transactions on Systems, Man, and Cybernetics Part B, 37(6), pp. 1512-1528.

[28] Armstrong-Helouvry, B., Dupont, P., and Canudas de Wit, C., 1994, “A survey of models, analysis tools and compensation methods for control of machines with friction," Automatica, 30 (6), pp. 1083-1138.

[29] Bona, B., and Indri, M., 2005, "Friction compensation in robotics: An overview", Proceedings of the 44th IEEE Conference on Decision and Control, and the European Control Conference, Seville, Spain, pp. $4360-4367$. 
Table 1: Parameters of the robots, friction terms and controllers

fig1. Block diagram of a general bilateral teleoperation system

fig2. Architecture of the proposed 4-channel adaptive teleoperation control approach

fig3. Revolute-prismatic robot

fig4. (a) Position tracking

fig4. (b) Position tracking error

fig4. (c) Force tracking

fig4. (d) Force tracking error

fig4. Fixed control scheme, which is not designed to deal with any uncertainties.

fig5. (a) Position tracking

fig5. (b) Position tracking error

fig5. (c) Force tracking

fig5. (d) Force tracking error

fig5. Conventional adaptive control, which merely deals with LP dynamic uncertainties.

fig6. (a) Position tracking

fig6. (b) Position tracking error

fig 6 (c) Force tracking

fig6. (d) Force tracking error

fig6. Proposed adaptive control, which deals with both LP and NLP dynamic uncertainties. 
Table 1: Parameters of the robots, friction terms and controllers

\begin{tabular}{cccccccc}
\hline \hline$m_{1}$ & $m_{2}$ & $l_{1}$ & $g$ & $\mu_{s i}$ & $\mu_{c i}$ & $v_{s i}$ & $\boldsymbol{\lambda}$ \\
$4.6 \mathrm{~kg}$ & $2.3 \mathrm{~kg}$ & $0.5 \mathrm{~m}$ & $9.8 \mathrm{kgm} / \mathrm{s}^{2}$ & $3.5 \mathrm{Nm}$ & $0.49 \mathrm{Nm}$ & $0.189 \mathrm{~s}^{2} / \mathrm{rad}^{2}$ & $0.1 \mathbf{I}$ \\
$\mathbf{K}_{m}$ & $\mathbf{K}_{s}$ & $\boldsymbol{\Gamma}_{\alpha m}$ & $\boldsymbol{\Gamma}_{\alpha s}$ & $\boldsymbol{\Gamma}_{\theta m}$ & $\boldsymbol{\Gamma}_{\theta s}$ & $\mathbf{C}_{2}$ & $\mathbf{C}_{3}$ \\
$10 \mathbf{I}$ & $10 \mathbf{I}$ & $0.05 \mathbf{I}$ & $0.05 \mathbf{I}$ & $0.05 \mathbf{I}$ & $0.05 \mathbf{I}$ & $300 \mathbf{I}$ & $300 \mathbf{I}$ \\
\hline \hline
\end{tabular}




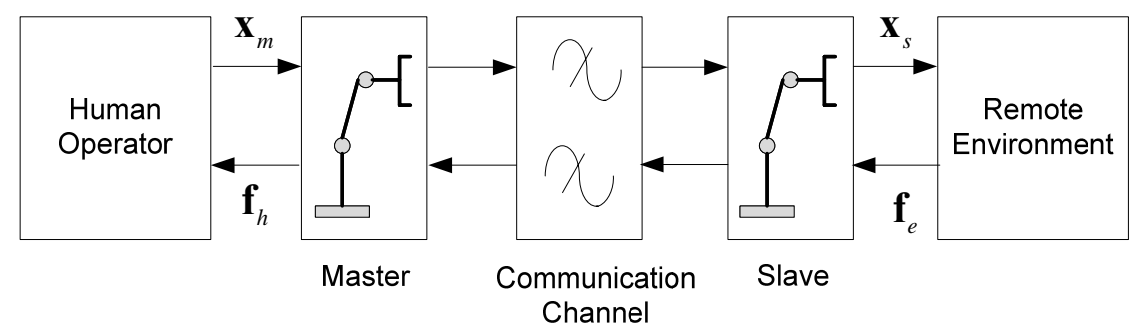

fig1. Block diagram of a general bilateral teleoperation system 


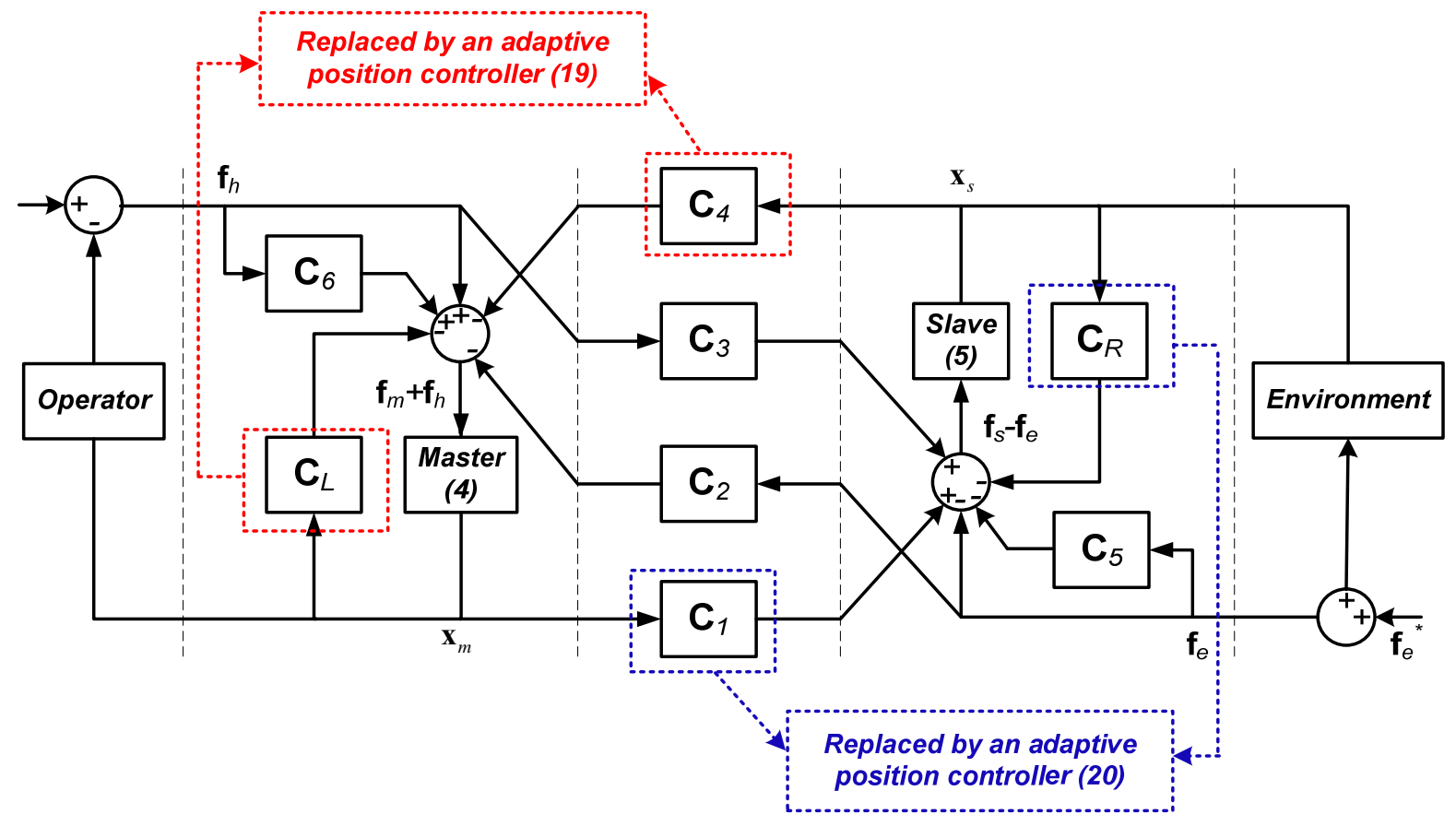

fig2. Architecture of the proposed 4-channel adaptive teleoperation control approach 


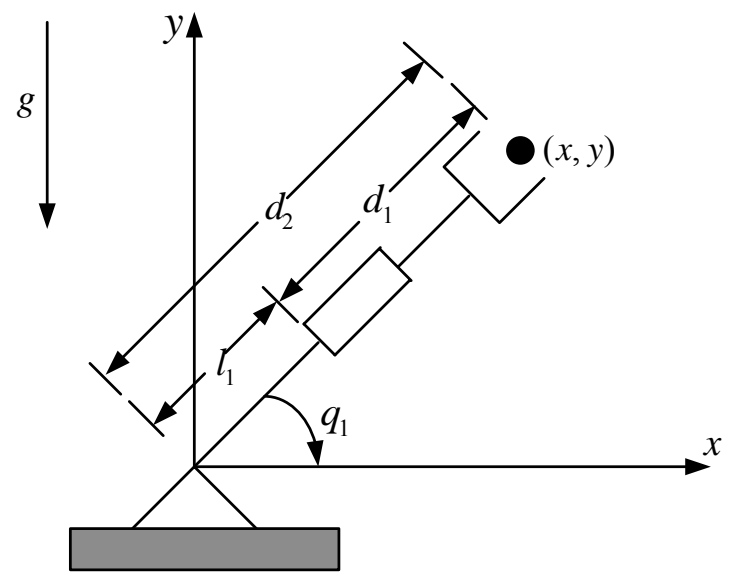

fig3. Revolute-prismatic robot

Xia Liu, DS-10-1274, 29 


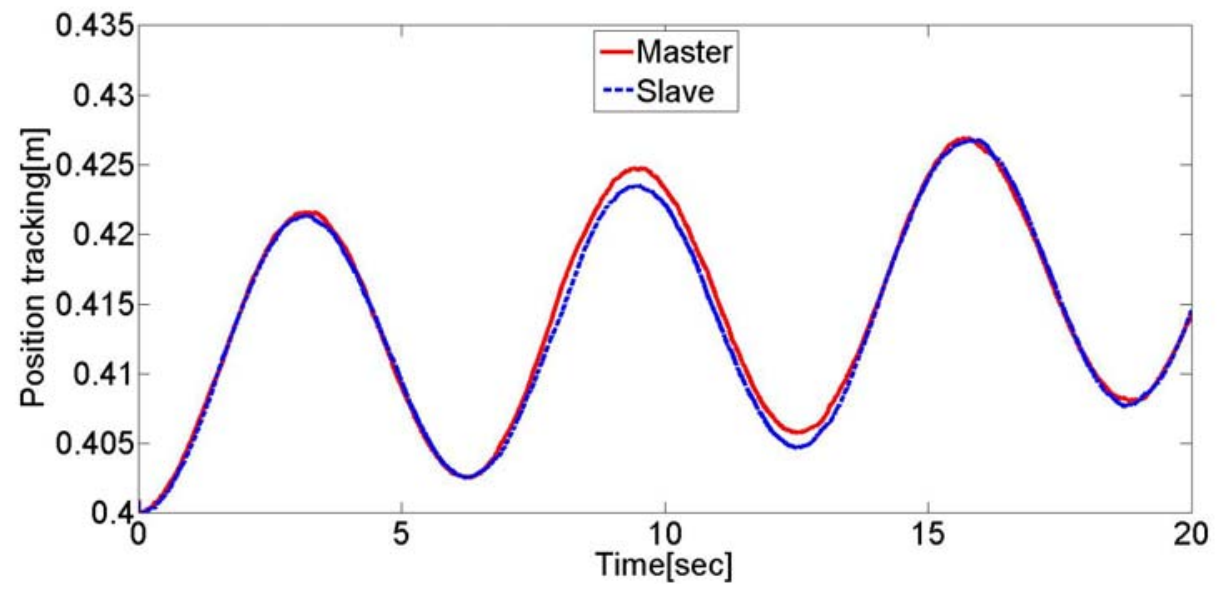

fig4. (a) Position tracking

Xia Liu, DS-10-1274, 30 


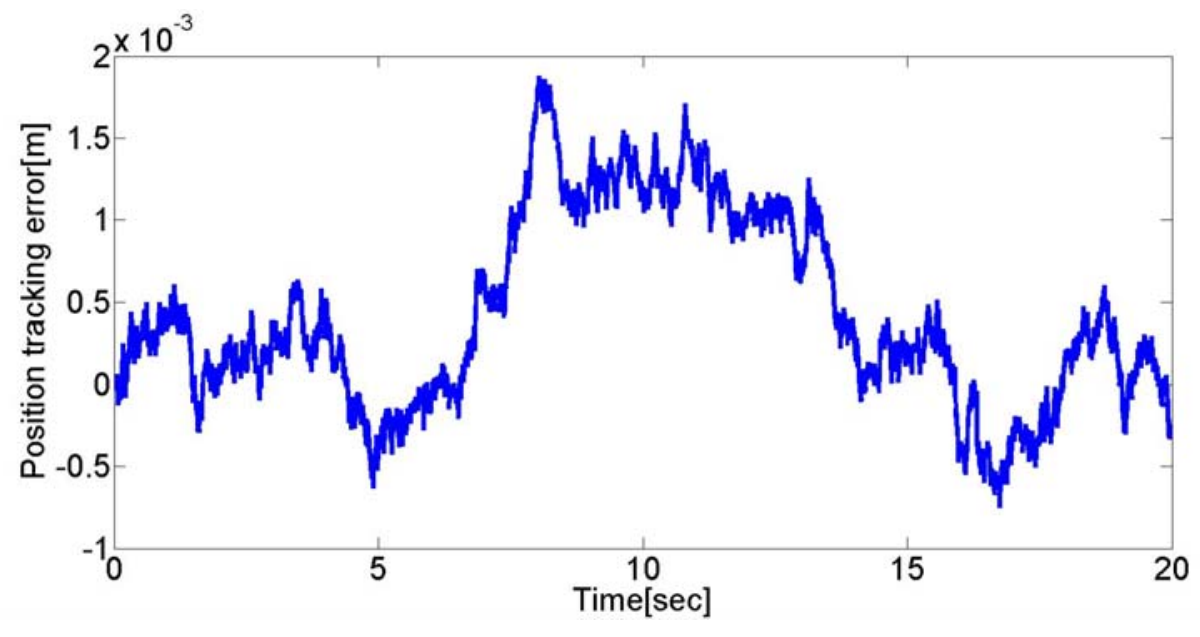

fig4. (b) Position tracking error 


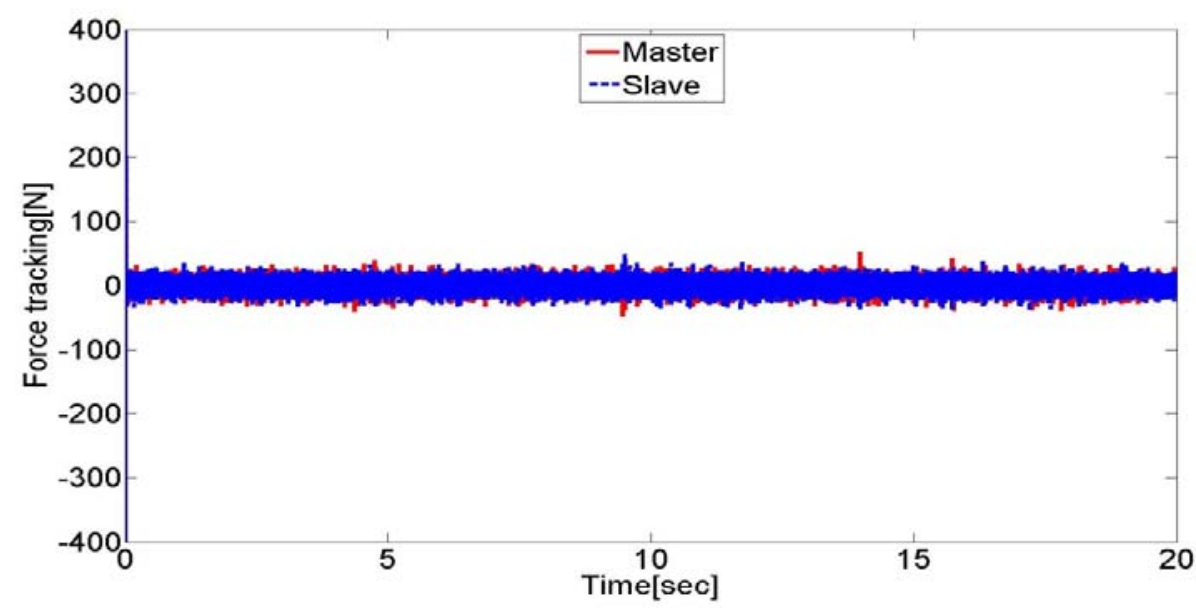

fig4. (c) Force tracking

Xia Liu, DS-10-1274, 32 


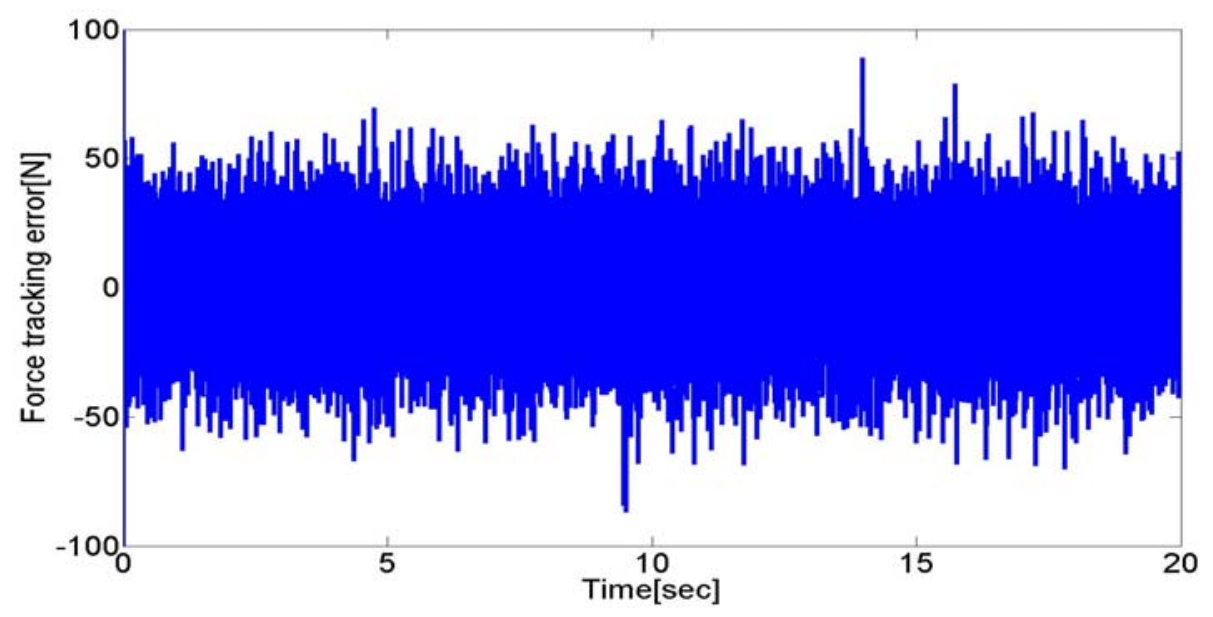

Fig. 4 (d) Force tracking error

fig4. Fixed control scheme, which is not designed to deal with any uncertainties. 


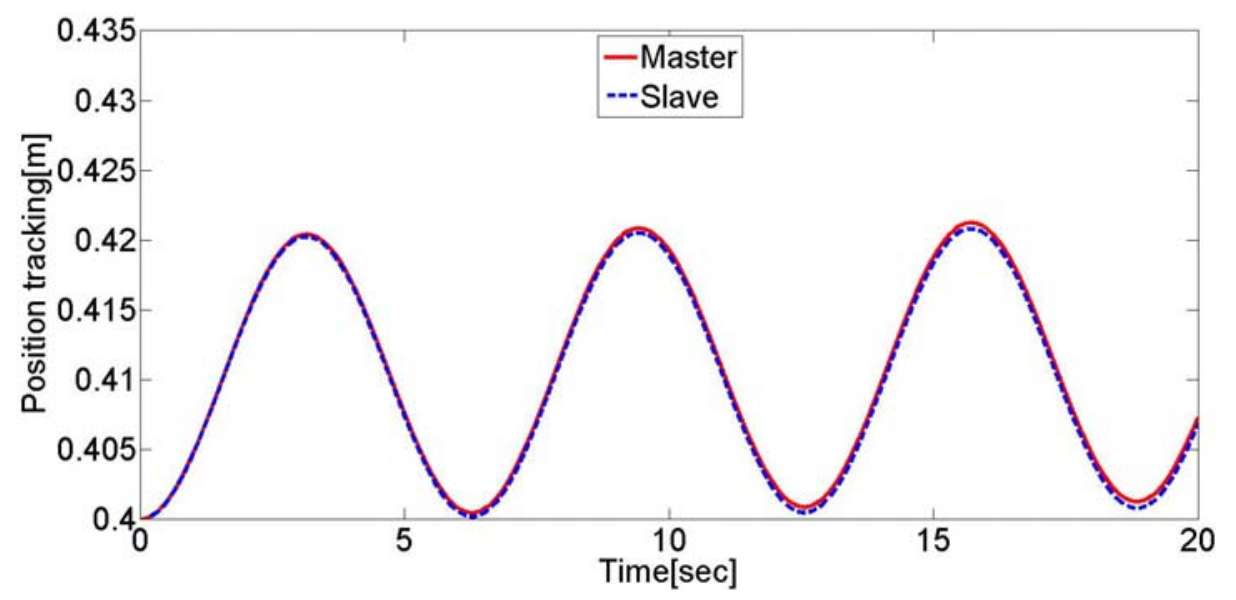

fig5. (a) Position tracking 


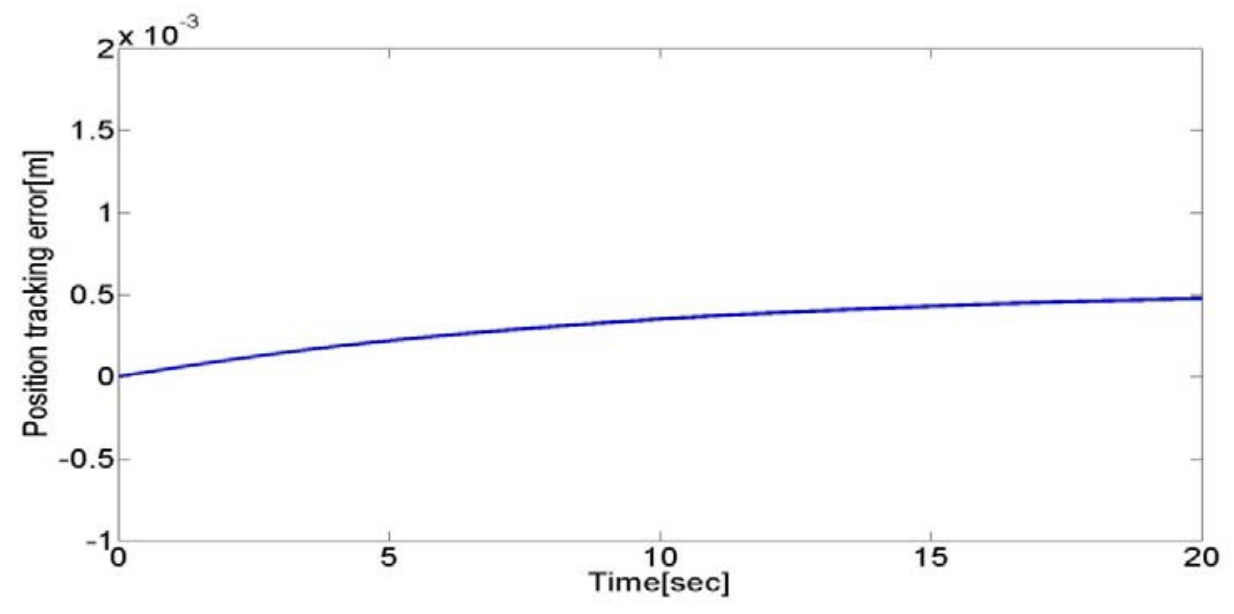

fig5. (b) Position tracking error

Xia Liu, DS-10-1274, 35 


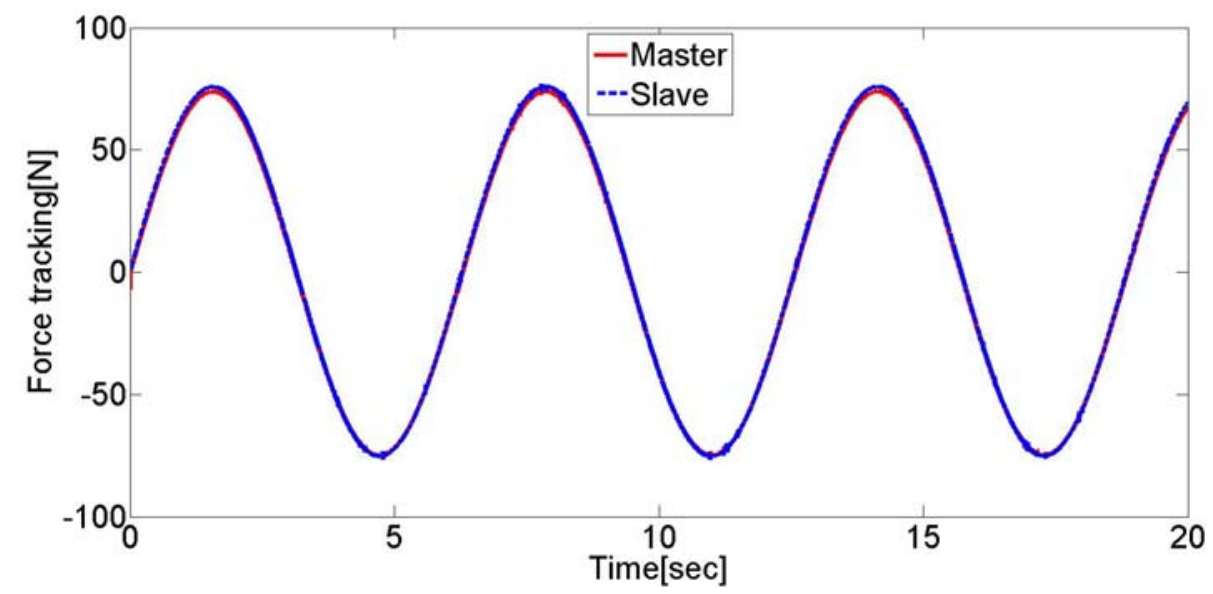

fig5. (c) Force tracking

Xia Liu, DS-10-1274, 36 


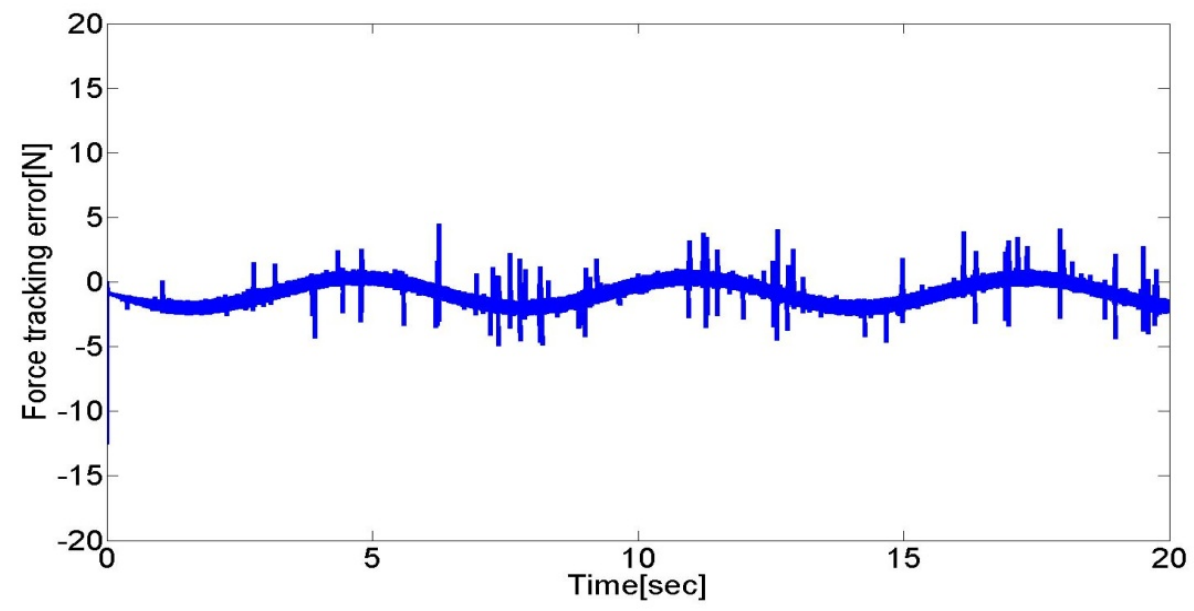

Fig. 5 (d) Force tracking error

fig5. Conventional adaptive control, which merely deals with LP dynamic uncertainties. 


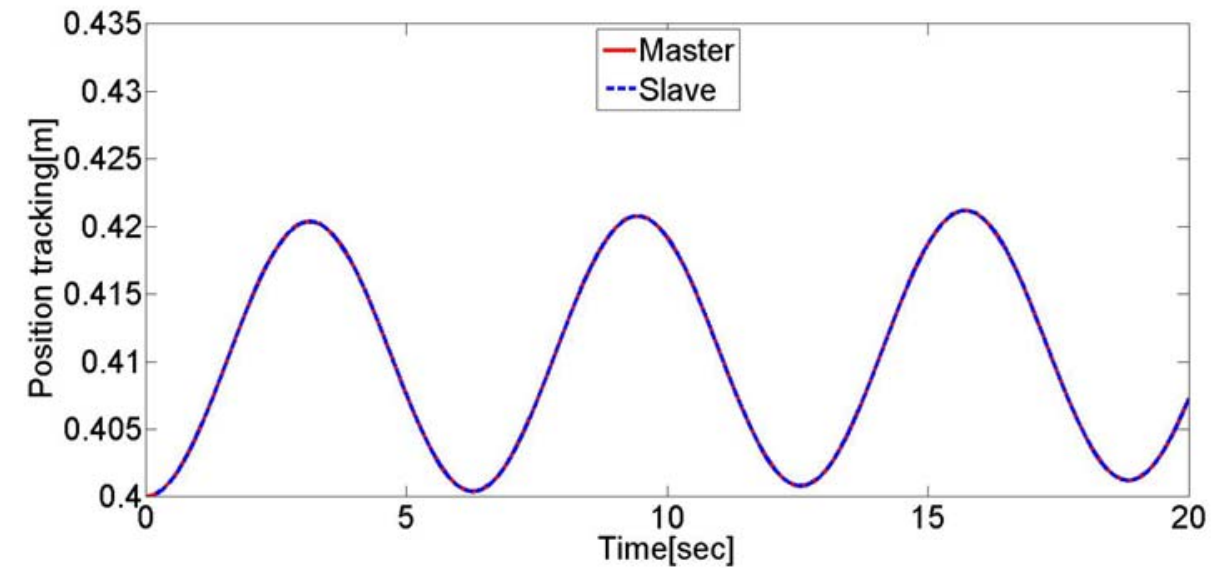

fig6. (a) Position tracking

Xia Liu, DS-10-1274, 38 


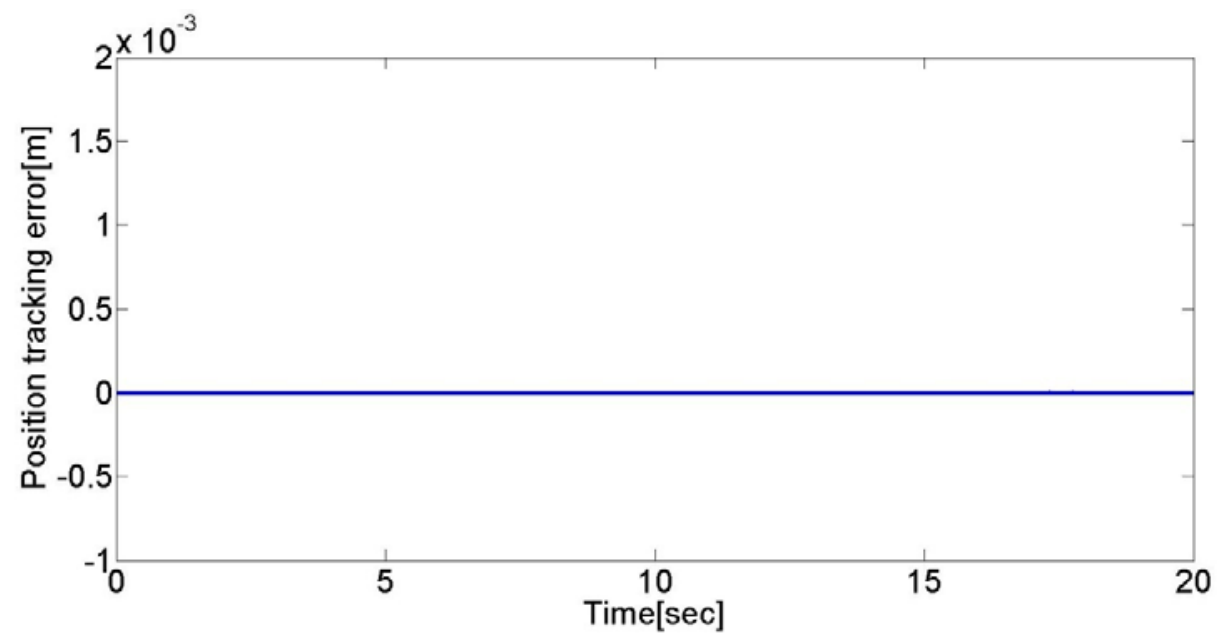

fig6. (b) Position tracking error

Xia Liu, DS-10-1274, 39 


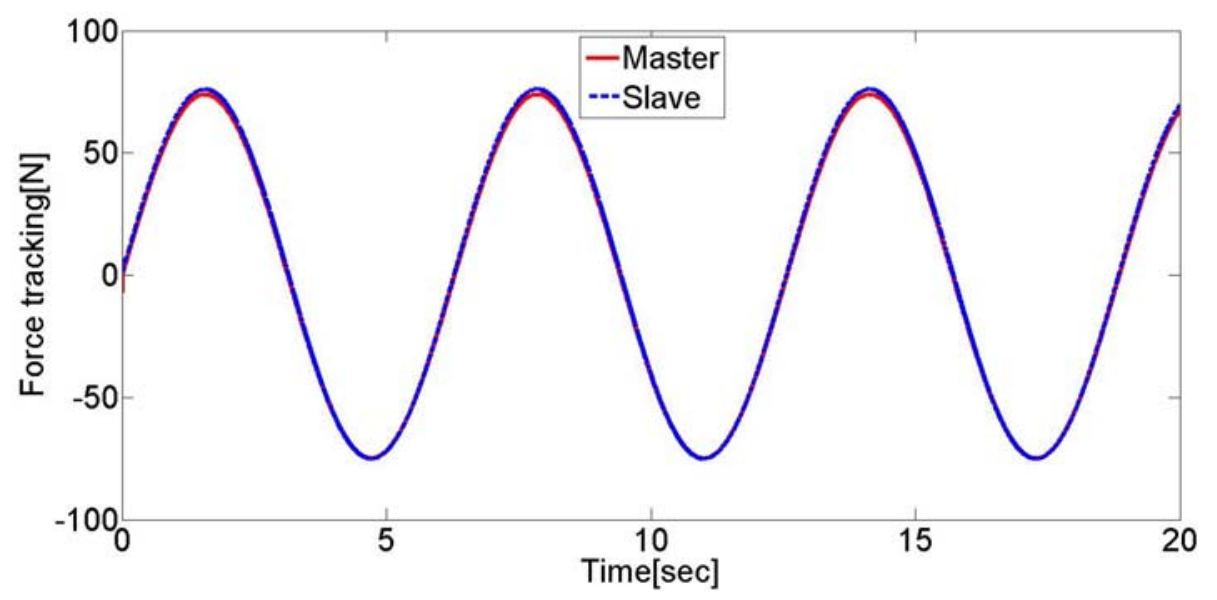

fig6. (c) Force tracking

Xia Liu, DS-10-1274, 40 


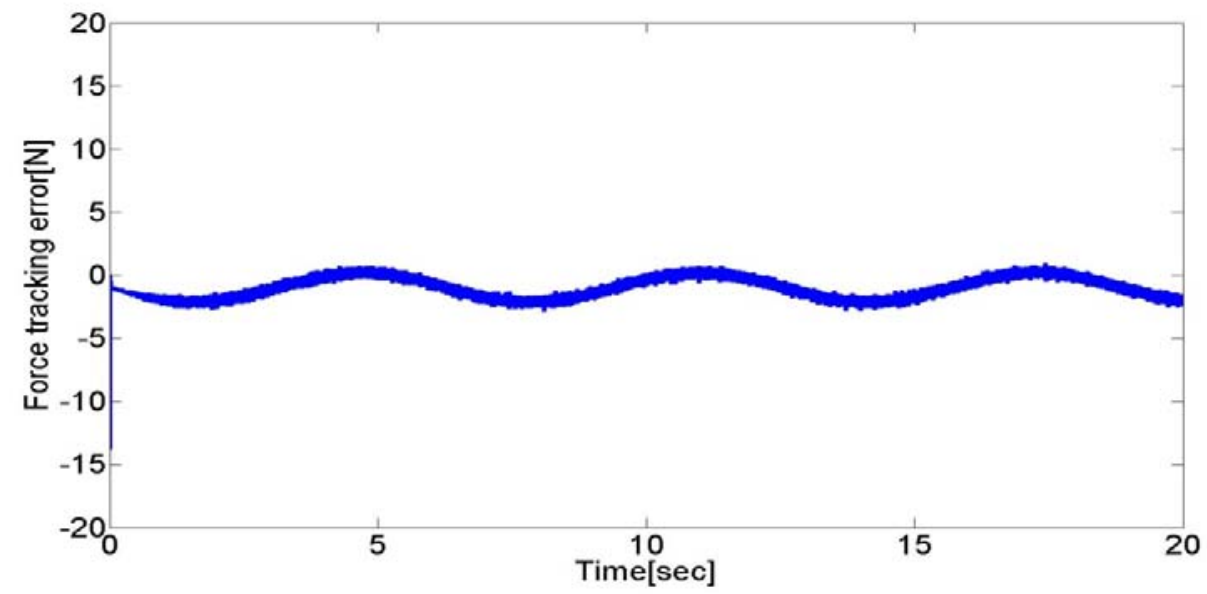

Fig. 6 (d) Force tracking error

fig6. Proposed adaptive control, which deals with both LP and NLP dynamic uncertainties. 